\title{
Theory and Design of Two-Parallelogram Filter Banks
}

\author{
Yuan-Pei Lin, Student Member, IEEE, and P. P. Vaidyanathan, Fellow, IEEE
}

\begin{abstract}
It is well known that the analysis and synthesis filters of orthonormal DFT filter banks can not have good frequency selectivity. The reason for this is that each of the analysis and synthesis filters have only one passband. Such frequency stacking (or configuration) in general does not allow alias cancelation when the individual filters have good stopband attenuation. A frequency stacking of this nature is called nonpermissible and should be avoided if good filters are desired. In a usual $M$ channel filter bank with real-coefficient filters, the analysis and synthesis filters have two passbands. It can be shown that the configuration is permissible in this case. Many designs proposed in the past demonstrate that filter banks with such configurations can have perfect reconstruction and good filters at the same time. In this paper, we develop the two-parallelogram filter banks, which is the class of 2-D filter banks in which the supports of the analysis and synthesis filters consist of two parallelograms. The two-parallelogram filter banks are analyzed from a pictorial viewpoint by exploiting the concept of permissibility. Based on this analysis, we construct and design a special type of twoparallelogram filter banks, namely, cosine-modulated filter banks (CMFB). In two-parallelogram CMFB, the analysis and synthesis filters are cosine-modulated versions of a prototype that has a parallelogram support. Necessary and sufficient conditions for perfect reconstruction of two-parallelogram CMFB will be derived in the paper.
\end{abstract}

\section{INTRODUCTION}

$\mathbf{T}$ HE $M$-channel filter bank has been studied extensively in the past (see Fig. 1 with scalar decimation ratio $M$ ). A 1-D $M$-band filter bank usually has the frequency stacking as shown in Fig. 2. The synthesis filters typically have the same passband regions as the corresponding analysis filters. A figure like this showing the passband regions of the filters will be called the support configuration of the filter bank. Filter banks with this type of configuration have been successfully designed through many approaches. It is possible to have perfect reconstruction and good individual analysis and synthesis filters (good frequency selectivity) at the same time. It turns out that the support configuration shown in Fig. 2 have two features that are necessary for a successful filter bank design. First, the support of each analysis filter does not overlap under modulo $2 \pi / M$. Filters with such a support are called aliasfree supported with respect to $M(\mathcal{A F S}(M))$. This means that if the filters are ideal, then their outputs allow aliasfree $M$ fold decimation, that is, no aliasing is created in the subbands.

Manuscript received March 7, 1995; revised May 14, 1996. This work was supported in part by NSF grant MIP 92-15785, Tektronix, Inc., and Rockwell International. The associate editor coordinating the review of this paper and approving it for publication was Dr. Bruce Suter.

The authors are with the Department of Electrical Engineering, California Institute of Technology, Pasadena, CA 91125 USA.

Publisher Item Identifier S 1053-587X(96)08236-0.

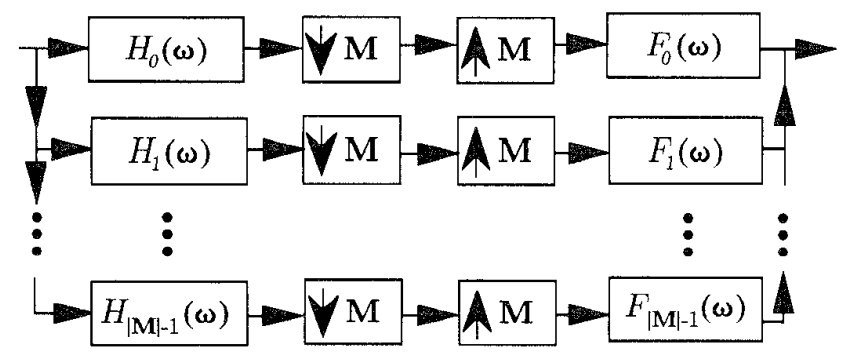

Fig. 1. $|M|$-channel maximally decimated filter bank, where $|M|$ denotes the absolute value of the determinant of $M$.

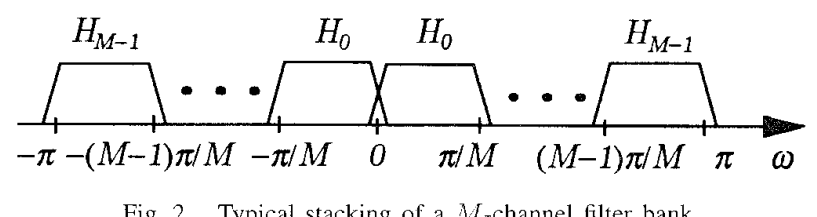

We say a configuration is $\mathcal{A} \mathcal{F} \mathcal{S}(M)$ if each analysis filter is $\mathcal{A F S}(M)$. The second feature of the support configuration in Fig. 2 is a property called permissibility. It is argued in [1] that with certain support configurations in a filter bank, a considerable amount of aliasing will remain uncanceled if the individual filters have good attenuation. In this case, the support configuration is called nonpermissible. The 1-D uniform DFT filter bank [2] is known to be an example of this nature. (The notion of configuration permissibility is more involved and will be explained in greater detail in Section II.) These two features are desirable for good filter bank design of any dimension.

Recently, there has been considerable interest in the design of 2-D maximally decimated filter banks (Fig. 1) [3]-[14]. For example, perfect reconstruction is achieved in [5] for a 2-D two-channel FIR filter bank with diamond-shaped filters. In [8], transformations are used to design higher dimensional filter banks from filter banks of lower dimensions. In [1], several issues regarding design of multidimensional filter bank are treated. In particular, the concept of support permissibility is introduced and discussed from a pictorial viewpoint. A study of a 2-D cosine modulated filter bank (CMFB) with rectangular-shaped but nonseparable prototype is made in [9]. The prototype of this type of 2-D CMFB has rectangular support but is allowed to be nonseparable. Nonseparable prototype with separable modulation is studied in [10]. In addition, 2-D nonseparable orthonormal wavelets using local cosine or sine bases are obtained in [11]-[13]. A nonseparable generalization 


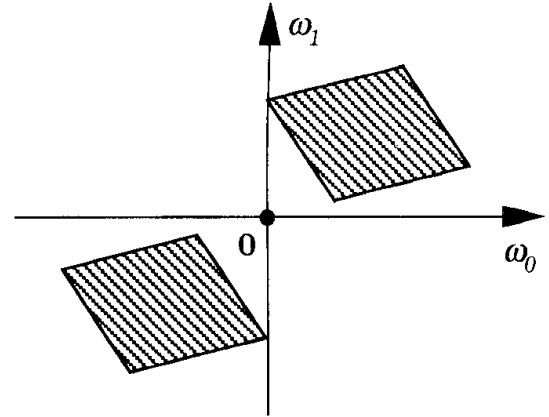

Fig. 3. Typical support of a two-parallelogram filter.

for 2-D CMFB is considered in [14]. However, the support of the 2-D filter banks studied therein is nonpermissible in general.

In the construction of 2-D filter banks, we have more variety in terms of possible configurations. Even if we impose the condition that all the analysis filters have real coefficients and have only two passbands, it is possible to have more than one configuration for a filter bank with decimation matrix $\boldsymbol{M}$. Various shapes can be used for the passbands of the individual filters, e.g., triangles [15] and parallelograms [16]. In this paper, we study the two-parallelogram filter banks, which are the class of 2-D filter banks in which the support of each analysis and synthesis filter is the union of two parallelograms (Fig. 3). Filters with this type of supports are called twoparallelogram filters.

\section{Two-Parallelogram Filter Banks}

A typical two-parallelogram filter bank has support configuration as shown in Fig. 4. This is a natural 2-D generalization of the frequency stacking in Fig. 2. For a two-parallelogram filter bank with decimation matrix $M$ as in Fig. 1, we will study the conditions such that the configuration is $\mathcal{A} \mathcal{F} \mathcal{S}(\boldsymbol{M})$. For this, we will derive the necessary and sufficient conditions such that a two-parallelogram support is $\mathcal{A F S}(\boldsymbol{M})$. For those $\mathcal{A} \mathcal{F} \mathcal{S}(\boldsymbol{M})$ configurations, we will further investigate permissibility of the configurations.

In this context of 1-D filter bank design, the cosinemodulated filter banks are well-known for low design cost and low complexity. The implementation of two-parallelogram filter banks using cosine-modulated filter banks yields similar advantage of economy. The two-parallelogram CMFB will be constructed and designed in this paper.

\section{Two-Parallelogram $C M F B$}

In two-parallelogram CMFB's, each of the two parallelograms of the analysis filters is a shifted version of a realcoefficient prototype, which has a parallelogram support and is, in general, nonseparable. Each analysis filter is a cosinemodulated version of the prototype, and each analysis filter consists of two copies of the prototype. The synthesis filters have the same spectral supports as the corresponding analysis filters. The analysis bank will eventually be constrained to be paraunitary; the analysis filter $H_{k}(\boldsymbol{\omega})$ and the corresponding synthesis filter $F_{k}(\boldsymbol{\omega})$ are related by $F_{k}(\boldsymbol{\omega})=H_{k}^{*}(\boldsymbol{\omega})$. All the

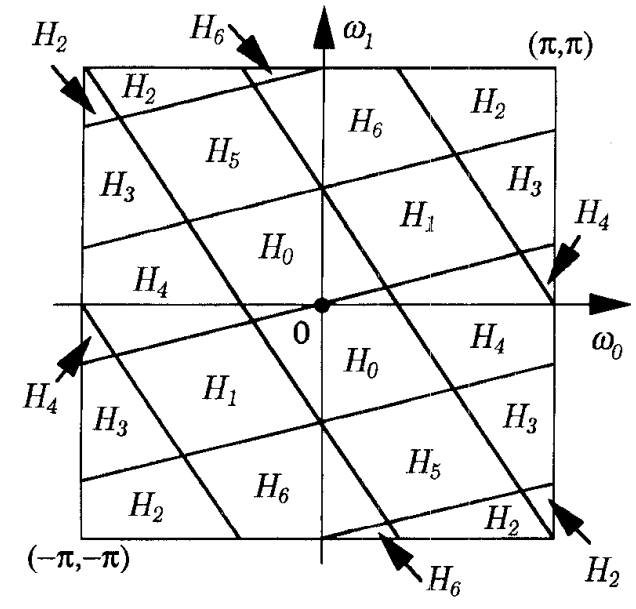

Fig. 4. Typical configuration of a two-parallelogram filter bank.

analysis and synthesis filters have real coefficients. We will present the sufficient conditions such that cancelation of major aliasing (due to overlapping transition bands) can be structurally enforced. Finally, having canceled the major aliasing, we constrain the prototype to ensure perfect reconstruction of the two-parallelogram CMFB.

We can conceive that in the more general case of 2-D filter banks the individual filters can have any even number of parallelogram. Particularly in the 2-D separable filter bank obtained by cascading 1-D filter banks, the support of each analysis filter consists of four rectangles. In a companion paper [17], we will study the four-parallelogram filter banks [18], which are the 2-D filter banks in which the supports of the analysis filters consist of four parallelograms. Note that two-parallelogram filter banks are fundamentally different from 2-D separable filter banks obtained from 1-D filter banks. However, four-parallelogram filter banks will reduce to separable 2-D filter bank in special cases.

Paper Outline: In Seciton II, we explain perfect reconstruction of 1-D filter banks from a pictorial viewpoint. This illustration will supply the explanation why $\mathcal{A F S}(\boldsymbol{M})$ property and permissible configurations are important for good design of analysis and synthesis filters. Section III is devoted to the study of two-parallelogram filter banks. For a successful design, the analysis filters should be $\mathcal{A F S}(\boldsymbol{M})$. Toward this end, we derive the necessary and sufficient conditions such that a two-parallelogram filter is $\mathcal{A} \mathcal{F} \mathcal{S}(\boldsymbol{M})$. Permissibility of the two-parallelogram filter bank will also be studied. Using the results developed in Section III, we construct $\mathcal{A} \mathcal{F} \mathcal{S}(\boldsymbol{M})$ configurations for the two-parallelogram CMFB (Section IV). In Section $\mathrm{V}$, the analysis and synthesis filters of two-parallelogram CMFB are formulated. The necessary and sufficient condition for the perfect reconstruction two-parallelogram CMFB is presented in Section VI. Efficient implementation and a design example of the two-parallelogram CMFB are given in Section VII. Preliminary versions of this work have been presented at international conferences [16], [19].

Preliminaries and Notations Notations in this paper are as in [2]. The fundamentals of integer matrices and 2-D multirate systems are employed frequently in this paper. A brief review 


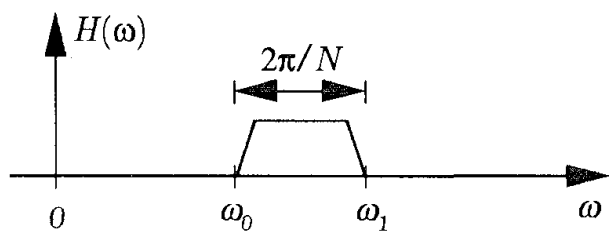

Fig. 5. Bandlimited signal with bandwidth $2 \pi / N$.

is given in Appendix A. Some of the frequently used notations are listed below:

1) Boldfaced lower-case letters are used to represent vectors, and boldfaced upper-case letters are reserved for matrices. The notations $A^{T}$ and $A^{\dagger}$ represent the transpose and transpose-conjugate of $\boldsymbol{A}$.

2) The Fourier transform of a 2-D signal $x(\boldsymbol{n})$ is denoted by $X(\boldsymbol{\omega})$, where $\omega$ is a $2 \times 1$ vector with $\boldsymbol{\omega}=\left[\begin{array}{ll}\omega_{0} & \omega_{1}\end{array}\right]^{T}$.

3) Vectors will also be used as subscript, e.g., $P_{\boldsymbol{k}}(\boldsymbol{\omega})$. If $k=\left[\begin{array}{ll}k_{0} & k_{1}\end{array}\right]^{T}$, then $P_{\boldsymbol{k}}(\omega)$, and $P_{\left(k_{0}, k_{1}\right)}(\boldsymbol{\omega})$ will be used interchangeably.

4) The notation $I$ denotes a $2 \times 2$ identity matrix.

5) If the support (passband) of a filter $H(\omega)$ does not overlap under modulo $2 \pi \boldsymbol{M}^{-T}, H(\omega)$ is called aliasfree supported with respect to $M(\mathcal{A F S}(\boldsymbol{M}))$. If, in addition to being $\mathcal{A} \mathcal{F} \mathcal{S}(\boldsymbol{M}), H(\boldsymbol{\omega})$ is also an ideal filter $(H(\boldsymbol{\omega})$ is 1 in the passband and 0 otherwise), then the output of $H(\omega)$ allows aliasfree $M$-fold decimation [20], [21], and in this case, $H(\boldsymbol{\omega})$ is called an aliasfree $(\boldsymbol{M})$ filter. A configuration is referred to as $\mathcal{A F S}(\boldsymbol{M})$ if all the analysis filters are $\mathcal{A} \mathcal{F} \mathcal{S}(\boldsymbol{M})$.

\section{BASIC CONSideration of Filter Bank Design}

In this section, we explain pictorially how perfect reconstruction is achieved for 1-D filter banks. The pictorial illustration will help us identify the roles of $\mathcal{A F S}(\boldsymbol{M})$ property and permissibility. To explain why these two features are important, we will use the CMFB's as an example. An $M$ band CMFB is a special case of $M$-band filter banks. It usually has the stacking in Fig. 2. The analysis and synthesis filters of CMFB's satisfy some additional properties. In particular, they are the cosine-modulated versions of a prototype filter. Although this property has made alias cancelation in CMFB's somewhat different from that in a usual filter bank, as the discussion proceeds, we will make observations for the more general filter banks. First, we review two 1-D sampling theorems and verify that the configuration in Fig. 2 is indeed $\mathcal{A} \mathcal{F} \mathcal{S}(M)$.

\section{One-Dimensional Sampling Theorems}

Recall the following two types of 1-D ideal filter $H(\omega)$ that is known to be aliasfree $(M)$.

Fact 2.1: Suppose $H(\omega)$ has bandwidth $2 \pi / N$ as shown Fig. 5; then, $H(\omega)$ is aliasfree $(M)$ if and only if $N \geq M$. The 2-D extension of this fact will be given in Section III. To compare with 2-D result to be stated later, we define $L=N / M$. In this case, $H(\omega)$ is aliasfree $(M)$ if and only if $L \geq 1$.

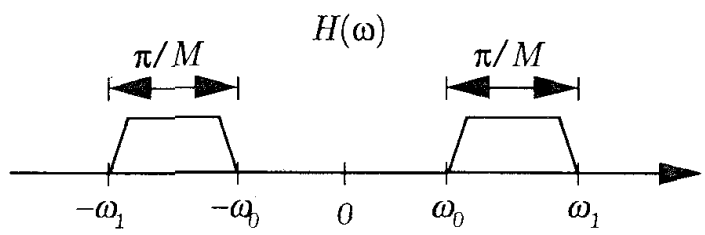

Fig. 6. Bandlimited real sequence with total bandwidth $2 \pi / M$

Fact 2.2-One-Dimensional Bandpass Sampling Theorem [22]: Suppose a 1-D ideal filter $H(\omega)$ has total bandwidth $2 \pi / M$ as shown in Fig. 6. Then, $H(\omega)$ is aliasfree $(M)$ if and only if $\omega_{0}$ is a multiple of $\pi / M$, i.e., $\omega_{0}=k \pi / M$, for some integer $k$.

In practice, the filters are not ideal but only $\mathcal{A F S}(M)$. The band edges of the analysis filters in Fig. 2 are multiples of $\pi / M$; the analysis filters are $\mathcal{A F S}(M)$. Therefore, the configuration in Fig. 2 is indeed $\mathcal{A F S}(M)$.

\section{One-Dimensional Cosine-Modulated Filter Banks (CMFB's)}

Two types of CMFB's have been developed: pseudo QMF systems [23]-[25] and perfect reconstruction systems [26]-[28]. Consider the $M$-channel filter bank in Fig. 1 (with scalar decimation ratio $M$ ). An $M$-channel CMFB (pseudoQMF or perfect reconstruction) is typically obtained by starting from a $2 M$-channel uniform DFT filter bank [2]. Each filter in the DFT filter bank is a shifted version of a lowpass prototype $P(\omega)$ (see Fig. 7(a)) with bandwidth $\pi / M$, which is half the total bandwidth of each filter in the desired $M$-channel system. The filters in the DFT filter bank are then shifted by $\pi / 2 M$ and paired to obtain real-coefficient analysis filters as in Fig. 7(b) for the $M$-channel CMFB. The shifts of the prototype are denoted by $P_{k}(\omega)$ in the figure. Each analysis filter has total bandwidth $2 \pi / M$, which is two times that of the prototype. In almost all the designs, the synthesis filters are time-reversed versions of the corresponding analysis filters; the analysis and synthesis filters have the same spectral support.

In the CMFB described above, as each analysis filter consists of two shifted copies of the prototype, each of the two copies has $M-1$ images due to decimation followed by expansion. Due to the $\mathcal{A} \mathcal{F S}(M)$ property, the images of the analysis filters are adjacent to the support of the corresponding synthesis filters but are not overlapping with the passbands of synthesis filters, as shown in Fig. 7(c). Thus, if the prototype filter is an ideal brick-wall filter, there is no aliasing, and the filter bank has perfect reconstruction. If the prototype filter is not ideal, those images that are adjacent to the synthesis filter result in major aliasing (Fig. 7(d)), whereas those that are not adjacent to the synthesis filters will be attenuated to the stopband level of the prototype filter. In the pseudo QMF CMFB, only the major aliasing errors are canceled, and approximate alias cancelation is attained. Approximate reconstruction is then achieved without sophisticated optimization of the lowpass prototype. In the perfect reconstruction CMFB, the prototype is optimized under further constraint (e.g., paraunitariness). The paraunitariness of the CMFB is guaranteed if the polyphase components of the prototype filter 


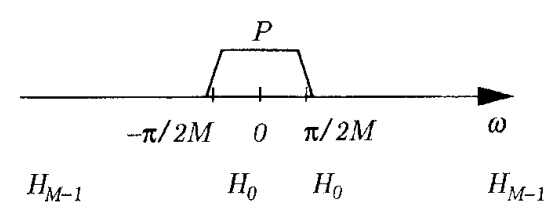

(a)

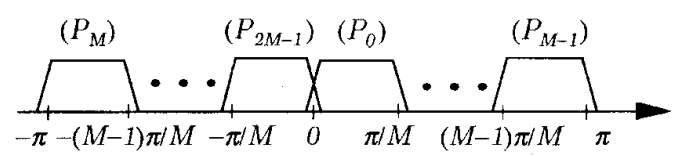

(b)

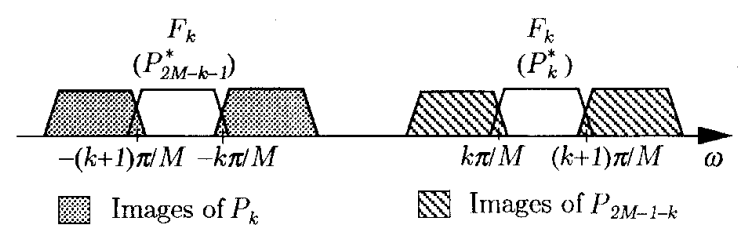

(c)

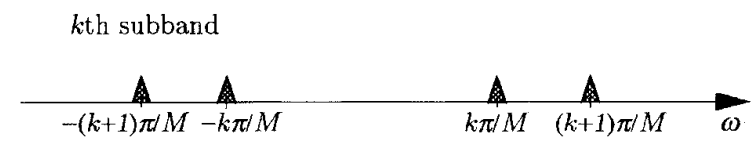

$(k+1)$ th subband

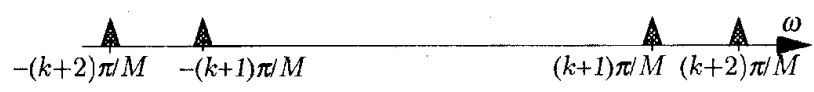

(d)

Fig. 7. One-dimensional cosine modulated filter bank. (a) Support of the prototype filter $P(\omega)$. (b) Support of the analysis filters $H_{k}(\omega)$. Each analysis filter has two parts, $P_{k}(\omega)$ and $P_{2} M-1-k(\omega)$. (c) Images of the analysis filter $H_{k}(\omega)$ that are adjacent to the synthesis filter $F_{k}(\omega)$. (d) Major aliasing in the $k$ th subband and the $(k+1)$ th subband.

satisfy some pairwise power complementary conditions [28]. In both pseudo QMF and perfect reconstruction systems, the design of the whole filter bank is reduced to the optimization of the lowpass prototype filter. The complexity of the analysis bank is equal to that of a prototype filter plus a DCT matrix.

\section{Main Features of the Configuration in Fig. 2}

From the preceding discussion, we observe that the support configuration in Fig. 2 has the following two features, which are necessary for designing a filter bank with good analysis and synthesis filters.

1) $\mathcal{A F S}(M)$ Property: Each analysis filter is $\mathcal{A} \mathcal{F S}(M)$, and the configuration is $\mathcal{A} \mathcal{F S}(M)$. This means that if the filters are ideal, they are aliasfree $(M)$ filters; no aliasing is created in the subbands, and the filter bank has perfect reconstruction. This feature is indispensable for the design of perfect reconstruction filter banks. For a configuration that is not $\mathcal{A} \mathcal{F} \mathcal{S}(M)$, severe aliasing will be created in the subbands, no matter how good the filters are. Without a $\mathcal{A} \mathcal{F} \mathcal{S}(\boldsymbol{M})$ configuration, a filter bank cannot have perfect reconstruction, even if the analysis and synthesis filters are ideal brick-wall filters.
2) Permissibility: From the discussion of CMFB, we see that major aliasing errors that contribute to the same aliasing transfer function $A_{i}(\omega)$ (defined in Appendix A) appear in pairs. For example, around the frequency $k \pi / M$ (Fig. 7(d)) both $k$ th and $(k+1)$ th subbands have major aliasing errors, and it can be verified that these two aliasing errors contribute to the same aliasing transfer function $A_{k}(\omega)$. This is essential if alias cancelation is to take place in CMFB. For the more general $M$-band filter banks, assume that the filters are not ideal but have good frequency selectivity. If a certain $A_{i}(\omega)$ has only one major aliasing term in a particular frequency region, this major aliasing cannot be canceled; perfect reconstruction is not possible. Therefore, if a perfect reconstruction filter bank has good analysis and synthesis filters, it is necessary that in any frequency region, there is more than one major aliasing term contributing to the same aliasing transfer function $A_{i}(\omega)$. A configuration without this feature will be referred to as nonpermissible. Permissibility allows the possibility of canceling major aliasing. If a filter bank has a nonpermissible configuration, the filters cannot have good stopband attenuation unless all the filters are ideal brick-wall filters. The 1-D uniform DFT filter bank [2] is known to be an example of this nature.

\section{Remarks on Permissibility}

1) The issue of permissibility arises only when nonideal filters are considered. In addition, permissibility is meaningful only when the underlying analysis and synthesis filters have frequency selectivity, i.e., when the notion of passbands and stopbands still makes sense. For example, in delay chain filter bank, the analysis and synthesis filters are allpass functions $\left(H_{k}(z)=z^{-k}\right.$ and $F_{k}(z)=$ $\left.z^{k}\right)$ and have no frequency selectivity. In this case, discussion of permissibility is meaningless.

2) To check the $\mathcal{A F S}(M)$ property of a configuration, we can individually examine each analysis filter. However, whether a configuration is permissible is determined jointly by all the analysis filters.

3) Permissibility is only a necessary condition for good filter bank designs. It does not suggest any constructive approach to design the filter banks.

\section{Two-PARALlElogram FILTER BANK}

In this section, we study a subclass of 2-D filter banks: the two-parallelogram filter banks. This is the class of filter banks in which the support of each analysis and synthesis filter is the union of two parallelograms. Before designing any filter bank, we first study the support configuration and see if good analysis and synthesis filters are possible. As a first step toward this, the analysis and synthesis filters should be $\mathcal{A F} \mathcal{S}(\boldsymbol{M})$ for a given decimation matrix $\boldsymbol{M}$. This calls for a bandpass sampling theorem for two-parallelogram filters. For those $\mathcal{A} \mathcal{F} \mathcal{S}(\boldsymbol{M})$ configurations, we further investigate permissibility of the configurations. 


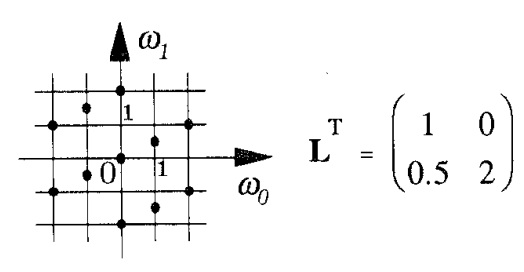

Fig. 8. Lattice of $L^{f}$.

\section{A. Sampling Theorems for One- and Two-Parallelogram Filters}

A 2-D filter $H(\boldsymbol{\omega})$ with a frequency support that consists of $k$ parallelograms is called a $k$-parallelogram filter. By definition, $H(\boldsymbol{\omega})$ is $\mathcal{A} \mathcal{F} \mathcal{S}(\boldsymbol{M})$ if the support of $H(\boldsymbol{\omega})$ does not overlap under modulo $2 \pi \boldsymbol{M}^{-T}$. However, for one- or twoparallelogram filters, there is no existing simple testing rule as those given in Facts 2.1 and 2.2. The 2-D equivalence of Facts 2.1 and 2.2 will be given in this subsection. Due to these 2-D extensions, we can easily test $\mathcal{A F S}(\boldsymbol{M})$ property of one- or two-parallelogram filters [29].

\section{One-Parallelogram $\mathcal{A} \mathcal{F} \mathcal{S}(M)$ Filters}

A result related to the multidimensional generalization of Fact 2.1 is as follows. Let $H(\boldsymbol{\omega})$ be a 2-D filter with support $S P D\left(\pi \boldsymbol{M}^{-T}\right)$ or a shifted version of $S P D\left(\pi \boldsymbol{M}^{-T}\right)$ for some integer matrix $\boldsymbol{M}$. When $H(\boldsymbol{\omega})$ has such a support, $H(\boldsymbol{\omega})$ is $\mathcal{A F S}(\boldsymbol{M})$ [2]. Now, consider the more general case that $H(\omega)$ is a one-parallelogram filter with support $\operatorname{SPD}\left(\pi \boldsymbol{N}^{-T}\right)$ or a shifted version of $\operatorname{SPD}\left(\pi \boldsymbol{N}^{-T}\right)$. The analysis of $\mathcal{A} \mathcal{F} \mathcal{S}(\boldsymbol{M})$ property of one-parallelogram filters is more intricate than that of 1-D one-passband case. Let us define

$$
L \triangleq M^{-1} N
$$

and denote the absolute value of the determinant of $L$ by $|L|$. The condition $|L| \geq 1$ alone does not imply the $\mathcal{A} \mathcal{F} \mathcal{S}(\boldsymbol{M})$ property [6], and a stronger condition is called for. In particular, the lattice of $L^{T}$ has to satisfy one additional property. A more precise statement is given in the theorem that follows.

Theorem 3.1: Let $H(\omega)$ be a one-parallelogram signal with frequency support $S P D\left(\pi N^{-T}\right)$ or a shifted version of $\operatorname{SPD}\left(\pi N^{-T}\right)$, where $N$ is possibly a noninteger matrix. Then, $H(\omega)$ is $\mathcal{A} \mathcal{F} \mathcal{S}(\boldsymbol{M})$ if and only if the matrix $L$ defined as $\boldsymbol{L}=\boldsymbol{M}^{-1} \boldsymbol{N}$ satisfies $\operatorname{LAT}\left(\boldsymbol{L}^{T}\right) \cap(-1,1)^{2}=\{\mathbf{0}\}$.

This necessary and sufficient condition means that no vector in $\operatorname{LAT}\left(\boldsymbol{L}^{T}\right)$ is inside the square $(-1,1)^{2}$ except the vector 0. For example, let $L^{T}=\left[\begin{array}{cc}1 & 0 \\ 0.5 & 2\end{array}\right]$. Then, $L A T\left(\boldsymbol{L}^{T}\right)$ is as shown in Fig. 8; $L A T\left(L^{T}\right)$ has only one vector (the vector 0 ) inside the square $(-1,1)^{2}$. Notice that in $1-D$ case, $|L| \geq 1$ if and only if $\operatorname{LAT}\left(L^{T}\right) \cap(-1,1)=\{0\}$. However, this relation does not hold in more than one dimension.

Proof of Theorem 3.1: Recall that $H(\omega)$ is $\mathcal{A F S}(M)$ if and only if the support of $H(\boldsymbol{\omega})$ does not overlap modulo $2 \pi \boldsymbol{M}^{-T}$. Therefore, $H(\boldsymbol{\omega})$ is $\mathcal{A} \mathcal{F} \mathcal{S}(\boldsymbol{M})$ if and only if, whenever $k_{1} \neq k_{2} \bmod M^{T}$,

$$
\begin{aligned}
\boldsymbol{\omega}_{1}- & 2 \pi \boldsymbol{M}^{-T} \boldsymbol{k}_{1} \\
& \neq \boldsymbol{\omega}_{2}-2 \pi \boldsymbol{M}^{-T} \boldsymbol{k}_{2}, \quad \forall \omega_{1}, \boldsymbol{\omega}_{2} \in \operatorname{SPD}\left(\pi \boldsymbol{N}^{-T}\right) .
\end{aligned}
$$

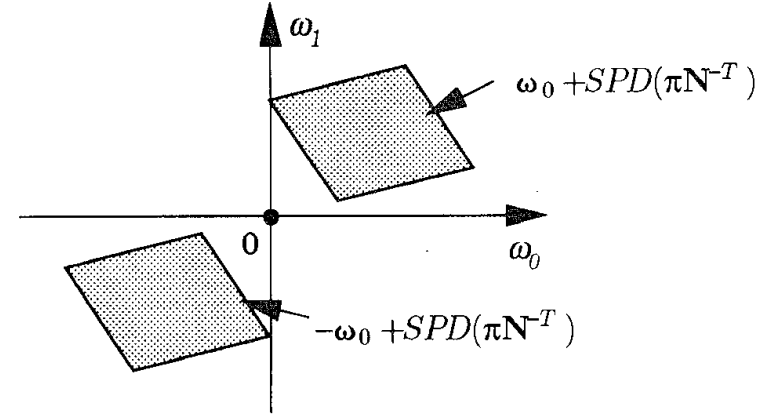

(a)

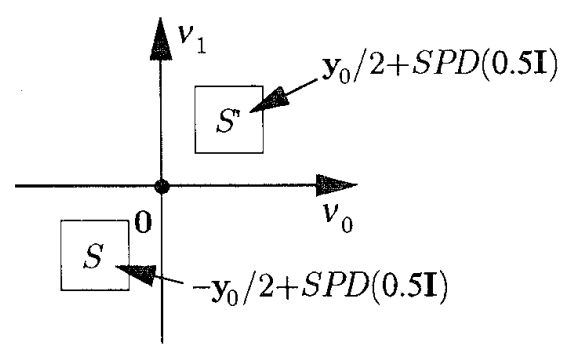

(b)

Fig. 9. (a) Support of a two-parallelogram filter $H(\omega)$. (b) Support of $H(\omega)$ with normalized axes.

Rearranging the above equation, we have $\omega_{1}-\omega_{2} \neq$ $2 \pi \boldsymbol{M}^{-T}\left(\boldsymbol{k}_{1}-\boldsymbol{k}_{2}\right)$. As $\boldsymbol{\omega}_{1}, \boldsymbol{\omega}_{2} \in \operatorname{SPD}\left(\pi \boldsymbol{N}^{-T}\right), \boldsymbol{\omega}_{i}$ can be expressed as $\omega_{i}=\pi \boldsymbol{N}^{-T} \boldsymbol{y}_{i}$ for some $2 \times 1$ vectors $\boldsymbol{y}_{i} \in[-1,1)^{2}, i=1,2$. Hence,

$$
\omega_{1}-\omega_{2}=2 \pi \boldsymbol{N}^{-T} \boldsymbol{y}, \quad \text { for some } \boldsymbol{y} \in(-1,1)^{2} .
$$

Using this expression, we have $y \neq L^{T}\left(k_{1}-k_{2}\right)$, for $k_{1} \neq$ $k_{2} \bmod M^{T}$. This is satisfied if and only if $\operatorname{LAT}\left(\boldsymbol{L}^{T}\right) \cap$ $(-1,1)^{2}=\{0\}$.

This theorem can be generalized for $D$-dimensional signals. The above technique of the proof can be carried out for signals of any dimensions.

\section{Bandpass Sampling Theorem for Two-Parallelogram Filters}

Now, consider the case that $H(\omega)$ is a two-parallelogram filter. The support of $H(\omega)$ (see Fig. 9) consists of two parallelograms, each a shifted version of $\operatorname{SPD}\left(\pi N^{-T}\right)$. The two passbands can be described as $\omega_{0}+S P D\left(\pi N^{-T}\right)$ and $-\omega_{0}+S P D\left(\pi \boldsymbol{N}^{-T}\right)$. Let $\boldsymbol{M}$ be an integer matrix with $|\boldsymbol{M}|=$ $|\boldsymbol{N}| / 2$. For $H(\boldsymbol{\omega})$ to be $\mathcal{A} \mathcal{F} \mathcal{S}(\boldsymbol{M})$, the 1-D bandpass sampling theorem suggests that the two parallelograms in the support of $H(\boldsymbol{\omega})$ should be properly located. On the other hand, the above sampling theorem for one-parallelogram signals indicates that in higher dimensions, the shape of the support also affects whether $\mathcal{A} \mathcal{F} \mathcal{S}(\boldsymbol{M})$ property is possible. Indeed, we will see that whether $H(\omega)$ is $\mathcal{A} \mathcal{F} \mathcal{S}(\boldsymbol{M})$ depends on the matrix $N$, as well as the relative position of the two parallelograms.

Theorem 3.2: Let $H(\omega)$ be a 2-D filter, and let the support of $H(\omega)$ be the union of two parallelograms, where each is a shifted version of $\operatorname{SPD}\left(\pi N^{-T}\right)$. The matrix $N$ is possibly a noninteger matrix. Let $\boldsymbol{M}$ be an integer matrix with $|\boldsymbol{M}|=$ 
$|\boldsymbol{N}| / 2$. Then, $H(\boldsymbol{\omega})$ is $\mathcal{A} \mathcal{F} \mathcal{S}(\boldsymbol{M})$ if and only if the following two conditions are satisfied:

1) Define $L \triangleq M^{-1} N$; then, $L^{T}$ has the form $L^{T}=\Gamma U$, where $U$ is a unimodular matrix, and $\Gamma$ is of one of the following forms:

$$
\left[\begin{array}{rr}
1 & \pm p \\
0 & 2
\end{array}\right],\left[\begin{array}{rr}
2 & 0 \\
\pm p & 1
\end{array}\right],\left[\begin{array}{rr}
2 & \pm p \\
0 & 1
\end{array}\right],\left[\begin{array}{rr}
1 & 0 \\
\pm p & 2
\end{array}\right], \quad 0<p \leq 1
$$

(a)

(c)

This is equivalent to saying that $|L|=2, L A T\left(\boldsymbol{L}^{T}\right) \cap$ $(-1,1)^{2}=\{0\}$ and $L^{T}$ has one integer row vector.

2) Let $\omega_{0}=\pi \boldsymbol{N}^{-T} \boldsymbol{y}_{0}$, where $\boldsymbol{y}_{0}$ is a $2 \times 1$ vector. Corresponding to the above four cases of $L, y_{0}$ satisfies

$$
\begin{aligned}
& {\left[\boldsymbol{y}_{0}\right]_{1} \text { is odd }\left[\boldsymbol{y}_{0}\right]_{0} \text { is odd } \boldsymbol{y}_{0}=\boldsymbol{L}^{T} \boldsymbol{k}+\left[\begin{array}{l}
1 \\
0
\end{array}\right]} \\
& \text { (a) } \\
& \boldsymbol{y}_{0}=\boldsymbol{L}^{T} \boldsymbol{k}+\left[\begin{array}{l}
0 \\
1
\end{array}\right], \quad \text { for some integer vector } \boldsymbol{k} \text {. }
\end{aligned}
$$

For example, let $L^{T}=\left[\begin{array}{cc}2 & 0 \\ 0.5 & 1\end{array}\right]$. One can verify that the first element of any vector $v \in L A T\left(\boldsymbol{L}^{T}\right)$ is an integer and that $\boldsymbol{L}$ satisfies the first condition. Notice the first condition is not necessary in a 1-D bandpass sampling theorem since $L=2$ in the 1-D case, and $L A T(L)$ consists of integers only. As indicated by the 1-D bandpass sampling theorem in Fact. 2.2, in 1-D case only the relative location of the two passbands needs to be constrained.

\section{Proof of Theorem 3.2}

We first show that these two conditions are necessary.

Condition 1 Is Necessary: An equivalent necessary and sufficient condition for $H(\omega)$ to be $\mathcal{A} \mathcal{F} \mathcal{S}(\boldsymbol{M})$ is that when $H(\boldsymbol{\omega})$ is decimated and then expanded by $M$, there is no overlapping in the passbands among $H(\boldsymbol{\omega})$ and the $|\boldsymbol{M}|-1$ images. For convenience, we will discuss the decimated and expanded version of $H(\boldsymbol{\omega})$. When $H(\boldsymbol{\omega})$ is decimated and then expanded by $\boldsymbol{M}$, each of the $|\boldsymbol{M}|-1$ images is a shifted version of $H(\boldsymbol{\omega})$; each consists of two parallelograms. For convenience, we normalize the frequency plane by $2 \pi N^{-T}$; the new axes $\nu_{0}$ and $\nu_{1}$ are the two entries of $\boldsymbol{\nu}=2 \pi \boldsymbol{N}^{-T} \boldsymbol{\omega}$. After normalization, the support of $H(\boldsymbol{\omega})$ appears to be the union of two squares (Fig. 9(b)), which are denoted by $S$ and $S^{\prime}$ with $S=-\boldsymbol{y}_{0} / 2+S P D(0.5 \boldsymbol{I})$ and $S^{\prime}=\boldsymbol{y}_{0} / 2+S P D(0.5 \boldsymbol{I})$. As $|\boldsymbol{N}|=2|\boldsymbol{M}|$, this is maximal decimation. Threfore, $H(\boldsymbol{\omega})$ and its $|\boldsymbol{M}|-1$ images fill the frequency plane; the normalized plane is tiled by the squares of $S P D(0.5 I)$. In a square tiling, we can observe at least one set of parallel lines (Fig. 10) and all the cells are bounded by these lines. For example, in the tiling of Fig. 10(a), we can observe one set of horizontal lines and all the squares are bounded by the horizontal lines. In Fig. 10(b), there is one set of vertical lines. Therefore, the images of passbands $S$ and $S^{\prime}$ are confined to these horizontal or vertical lines. Suppose the images of $S$ are located at $-\boldsymbol{y}_{0} / 2+c$ for some vector $c$.

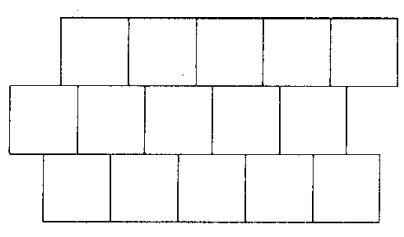

(a)

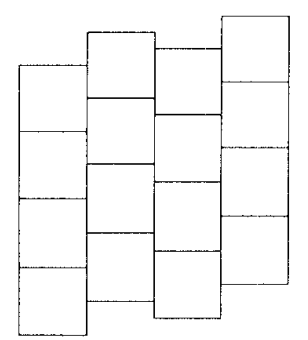

(b)

Fig. 10. Square tiling with (a) horizontal lines and (b) vertical lines.

Then, $[c]_{1}$ must be an integer when the images are bounded by horizontal lines, and $[\boldsymbol{c}]_{0}$ must be an integer when the images are bounded by vertical lines. On the other hand, observe that the images of $S$ are located at $-\boldsymbol{y}_{0} / 2+L A T\left(\boldsymbol{L}^{T}\right) \boldsymbol{k}$ for some integer vector $k$. To have the images of $S$ located between the horizontal or vertical lines, $L^{T}$ is necessarily of the form

$$
\left[\begin{array}{cc}
d_{0} & d_{1} \\
\times & \times
\end{array}\right] \text { or }\left[\begin{array}{cc}
\times & \times \\
d_{0} & d_{1}
\end{array}\right]
$$

for some integers $d_{0}$ and $d_{1}$, i.e., $\boldsymbol{L}^{T}$ has one integer row.

Notice that if a two-parallelogram filter with parallelogram prototype $\operatorname{SPD}\left(\pi \boldsymbol{N}^{-T}\right)$ is $\mathcal{A F} \mathcal{S}(\boldsymbol{M})$, then a one-parallelogram filter with support $\operatorname{SPD}\left(\pi \boldsymbol{N}^{-T}\right)$ is also $\mathcal{A F S}(\boldsymbol{M})$. For this, $\boldsymbol{L}$ must satisfy $\operatorname{LAT}\left(\boldsymbol{L}^{T}\right) \cap(-1,1)^{2}$. Combining this condition and the fact that $|L|=2$, after some row operation, we can arrive at (1) from (3). Conversely, if $L$ is of the forms in (1), then we can verify that $|\boldsymbol{L}|=2, \operatorname{LAT}\left(\boldsymbol{L}^{T}\right) \cap(-1,1)^{2}$, and $\boldsymbol{L}^{T}$ has one integer row vector. Corresponding to the four cases in (1), the passband $S$ and its images are as shown in Fig. 11 with $p=0.25$. Fig. 11(e) shows that case when $L$ is as in (c) of (1) with $p=1$.

Condition 2 Is Necessary: To satisfy the bandpass sampling theorem, the other passband $S^{\prime}$ must be located in one of the lighter shaded cells that are not occupied yet. In the first case (Fig. 11(a)), the second passband can be located anywhere in the lighter shaded stripe; $\left[\boldsymbol{y}_{0}\right]_{1}$ is an odd integer. In the third case (Fig. 11(c)), lighter shaded cells can be described as

$$
\begin{gathered}
-\boldsymbol{y}_{0} / 2+\left(\boldsymbol{L}^{T} \boldsymbol{k}+\left[\begin{array}{l}
1 \\
0
\end{array}\right]\right)+S P D(0.5 \boldsymbol{I}), \\
\text { where } \boldsymbol{k} \text { is an integer vector. }
\end{gathered}
$$

When the passband $S^{\prime}$ is in one of the lighter shaded cells $\boldsymbol{y}_{0} / 2=-\boldsymbol{y}_{0} / 2+\left(\boldsymbol{L}^{T} \boldsymbol{k}+\left[\begin{array}{l}1 \\ 0\end{array}\right]\right)$. Therefore, $\boldsymbol{y}_{0}=\boldsymbol{L}^{T} \boldsymbol{k}+\left[\begin{array}{l}1 \\ 0\end{array}\right]$. Similarly, we can verify the second and the fourth cases.

Conversely, if $L^{T}$ is of the form in (1) and $y_{0}$ is given as in (2), we can verify that the passbands of $H(\boldsymbol{\omega})$ and the 


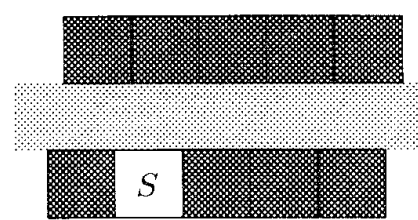

(a)

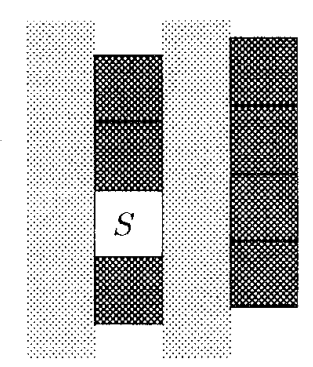

(b)

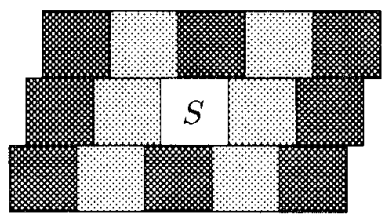

(c)

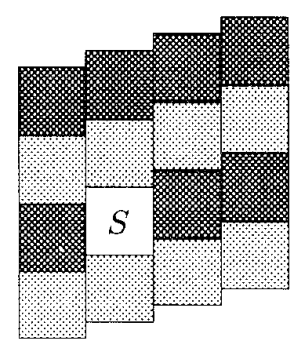

(d)

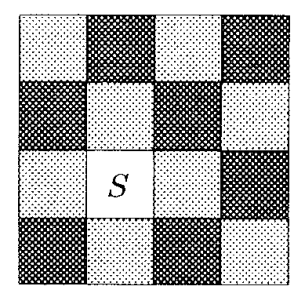

(e)

Fig. 11. Passband $S$ and relative position to its images (darker shaded squares) for various cases of $L A T\left(L^{t}\right)$

images are properly interlaced and that the bandpass sampling theorem is satisfied.

\section{Remarks}

1) We would like to point out one necessary condition implied by Theorem 3.2. The vector $\boldsymbol{y}_{0}$ has at least one nonzero integer entry. The importance of this necessary condition will be observed in the next remark.

2) Continuous Time Maximal Decimation: Let $H(\Omega)$ be the Fourier transform of a 2-D continuous time filter $h(\boldsymbol{t})$.

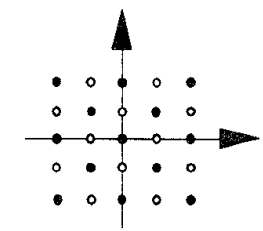

Fig. 12. Quincunx lattice.

Suppose $H(\Omega)$ is an ideal filter and that the support of $H(\Omega)$ is the union of two parallelograms, where each is a shifted version of $S P D\left(\pi N^{-T}\right)$, and the two parallelograms are separated by $2 \pi \boldsymbol{N}^{-T} \boldsymbol{y}_{0}$. The question is as follows: What are the conditions such that the output of $H(\Omega)$ can be maximally decimated? The necessary and sufficient condition for this is that $\boldsymbol{y}_{0}$ has at least one nonzero integer entry. As long as $H(\Omega)$ satisfies this condition, we can always find $M$ with $|\boldsymbol{M}|=|\boldsymbol{N}| / 2$ such that $H(\Omega)$ is aliasfree $(\boldsymbol{M})$. Therefore, maximal aliasfree decimation of the output of $H(\Omega)$ depends entirely on the relative position of the two passbands. However, in the 1-D case, the condition that $y_{0}$ is a nonzero integer is not sufficient. The necessary and sufficient condition in 1-D case is that $y_{0}$ is an odd integer.

\section{Properties of two-Parallelogram Filters}

In all cases of Fig. 11, we observe that $S$ is adjacent to its own images. This is, in general, true; it can be verified that $S$ is necessarily adjacent to its own images when the bandpass sampling theorem is satisfied. The type of adjacency is determined by $\operatorname{LAT}\left(\boldsymbol{L}^{T}\right)$. More specifically, we have the following three cases:

1) Complete Edge Adjacency: When $L$ is as in (a) or (b) of (1), $S$ is adjacent to its images on two edges; $S$ and its images form a stripe pattern (Fig. 11(a)-(b)).

2) Partial Edge Adjacency: When $L$ is as in (c) or (d) of (1) with $p \neq 1, S$ is partially edge adjacent to its images (Fig. 11(c)-(d))

3) Vertex Adjacency: When $L$ is as in (c) or (d) of (1) with $p=1, \operatorname{LAT}\left(\boldsymbol{L}^{T}\right)$ is quincunx (Fig. 12). In this case, $S$ is vertex adjacent to its images; $S$ and its images form a check pattern (Fig. 11(e)). Notice that this is very different from the 1-D case. Recall that in the 1-D case, no passbands of the analysis filters are adjacent to their own images. This observation will help us to study the permissibility of two-parallelogram filter banks.

\section{B. Permissibility of Two-Parallelogram Filter Banks}

Now, consider a two-parallelogram filter bank. In the subbands, the images of the analysis filters will be attenuated to the stopband level of the synthesis filter, except those images that are adjacent to the synthesis filters. Those adjacent images result in major aliasing if the individual filters are assumed to have good frequency selectivity. In the 2-D case, there are several different types of adjacency, which result in different types of major aliasing. As the notion of 


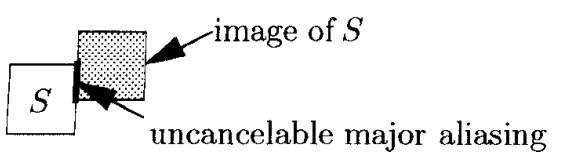

Fig. 13. Illustration of uncancelable major aliasing.

permissibility originates from uncancelable major aliasing, we have to consider a finer classification of permissibility. Consider the different cases in Fig. 11. Suppose the shaded areas represent the images of $S$ or $S^{\prime}$. In all cases, one image is adjacent to $S$ and will result in different major aliasing. For example, in Fig. 11(a)-(d), the image results in edge aliasing, whereas in Fig. 11(e), the image results in vertex aliasing. For an alias transfer function $A_{\boldsymbol{k}}(\boldsymbol{\omega})$, if in a certain frequency region there is only one particular major aliasing term, then this major aliasing cannot be canceled. In this case, when the uncancelable aliasing is edge based or vertex based, the support is called edge nonpermissible or vertex nonpermissible, respectively. We see that edge adjacency corresponds to band adjacency in the 1-D case (Fig. 7), whereas vertex adjacency has no 1-D correspondence. Comparing these two types of nonpermissibility, edge-based aliasing is, in general, much more serious than vertex based. The filter banks that do not have any type of uncancelable major aliasing is called permissible. We will explain below that the two-parallelogram filter banks can have edge permissible but cannot have both edge and vertex permissibility.

When the images of $S$ are adjacent to $S$, major aliasing is created and this major aliasing is uncancelable. To explain this, suppose $L^{T}=\left[\begin{array}{ll}1 & 0 \\ p & 2\end{array}\right]$, as in (d) of (1). As the vector $\left[\begin{array}{ll}1 & p\end{array}\right]^{T} \in L A T\left(\boldsymbol{L}^{T}\right)$, one image of $S$ will be separated from $S$ by $\left[\begin{array}{ll}1 & p\end{array}\right]^{T}$. This image is edge adjacent to $S$ and creates major aliasing as in Fig. 13. The resulting major aliasing contributes to the alias transfer function $A_{(1,0)}(\boldsymbol{\omega})$ since $\left[\begin{array}{ll}1 & p\end{array}\right]^{T}=L^{T}\left[\begin{array}{ll}1 & 0\end{array}\right]^{T}$. In other subbands, the major aliasing errors that contribute to $A_{(1,0)}(\omega)$ are not in the the same frequency region; this major aliasing is uncancelable. However, from previous subsection, we know images of $S$ will always be adjacent to $S$. We conclude that two-parallelogram filter banks are not permissible in general under the assumption of good analysis and synthesis filters.

On the other hand, in Fig. 11(e), we observe that images of $S$ are only vertex adjacent to $S$. Therefore, edge permissibility is possible. The two-parallelogram CMFB's that have edge permissibility will be constructed in the next section.

\section{Configuration of Two-Parallelogram CMFB's}

In view of the construction procedure for 1-D CMFB's in Section II and the discussion in previous section, there are three important issues to be addressed. First, for a given filter bank with decimation matrix $M$ as in Fig. 1, we ask how to obtain the analysis filters such that the support configuration of the 2-D CMFB is an extension of 1-D version. The second issue is the analysis and synthesis filters should be $\mathcal{A F S}(\boldsymbol{M})$, and the configuration should be $\mathcal{A} \mathcal{F} \mathcal{S}(\boldsymbol{M})$. Moreover, as the supports of the analysis filters form a tiling of the frequency plane, the filter bank has perfect reconstruction when the prototype filter is an ideal filter. For those that satisfy these two criteria, we further consider support permissibility.

\section{Issue 1-Support Configuration}

The general setting of 1-D $M$-channel CMFB can be summarized as follows:

1) Design a $2 M$-channel uniform DFT filter bank.

2) Shift the filters in the DFT filter bank by $\pi / 2 M$, and combine appropriate pairs of filters to yield realcoefficient analysis filters.

We now translate these procedures to the 2-D case and construct the two-parallelogram CMFB. To design a twoparallelogram CMFB with decimation matrix $M$, conceptually, we start from a uniform DFT filter bank [2] with decimation matrix $N=M L$, where $L$ is an integer matrix (to be chosen appropriately) with $|\boldsymbol{L}|=2$. For example,

$$
\text { let } \begin{aligned}
\boldsymbol{M} & =\left[\begin{array}{cc}
7 & -2 \\
0 & 1
\end{array}\right] \text { and } \boldsymbol{L}=\left[\begin{array}{ll}
1 & 1 \\
2 & 4
\end{array}\right], \quad \text { then } \\
\boldsymbol{N} & =\left[\begin{array}{rr}
3 & -1 \\
2 & 4
\end{array}\right]
\end{aligned}
$$

As $|N|=14$, there are a total of 14 DFT filters as shown in Fig. 14. The DFT filters $P_{i}(\boldsymbol{\omega})$ are given by

$$
P_{i}(\boldsymbol{\omega})=P_{\mathbf{0}}\left(\boldsymbol{\omega}-2 \pi \boldsymbol{N}^{-T} \boldsymbol{k}_{i}\right), \quad \boldsymbol{k}_{i} \in \mathcal{N}\left(\boldsymbol{N}^{T}\right) .
$$

Each filter in the DFT filter bank is a shifted version of a prototype $P(\omega)$, which has a parallelogram support $S P D\left(\pi \boldsymbol{N}^{-T}\right)$. For a given $M$, the support of the prototype is different for different choice of $\boldsymbol{L}$. In 1-D CMFB, we shift the filters by $\pi / 2 M$, but in $2-\mathrm{D}$ case, the shifts are vector shifts, and we can shift the filters by

$$
\pi \boldsymbol{N}^{-T}\left[\begin{array}{ll}
1 & 0
\end{array}\right]^{T}, \quad \pi \boldsymbol{N}^{-T}\left[\begin{array}{ll}
0 & 1
\end{array}\right]^{T}, \quad \text { or } \quad \pi \boldsymbol{N}^{-T}\left[\begin{array}{ll}
1 & 1
\end{array}\right]^{T},
$$

as shown in Fig. 15. Fig. 16(a)-(c) shows the results with respect to the three shifts. The filters $Q_{A, i}(\boldsymbol{\omega}), Q_{B, i}(\boldsymbol{\omega})$ and $Q_{C, i}(\omega)$ are given by

$$
\begin{aligned}
& Q_{A, i}(\boldsymbol{\omega})=P_{i}\left(\boldsymbol{\omega}-2 \pi \boldsymbol{N}^{-T}\left[\begin{array}{ll}
0.5 & 0
\end{array}\right]^{T}\right), \\
& Q_{B, i}(\boldsymbol{\omega})=P_{i}\left(\boldsymbol{\omega}-2 \pi \boldsymbol{N}^{-T}\left[\begin{array}{ll}
0 & 0.5
\end{array}\right]^{T}\right), \\
& Q_{C, i}(\boldsymbol{\omega})=P_{i}\left(\boldsymbol{\omega}-2 \pi \boldsymbol{N}^{-T}\left[\begin{array}{ll}
0.5 & 0.5
\end{array}\right]^{T}\right) .
\end{aligned}
$$

For all the three cases, filters can be paired to obtain realcoefficient analysis filters. For example, in Fig. 16(a), the filter coefficients of $Q_{A, i}(\boldsymbol{\omega})$ and $Q_{A, i}^{\prime}(\boldsymbol{\omega})$ are complex conjugates of each other. The filters $Q_{A, i}(\boldsymbol{\omega})$ and $Q_{A, i}^{\prime}(\boldsymbol{\omega})$ can be paired to obtain the analysis filter $H_{A, i}(\boldsymbol{\omega})$

$$
H_{A, i}(\boldsymbol{\omega})=Q_{A, i}(\boldsymbol{\omega})+Q_{A, i}^{\prime}(\boldsymbol{\omega})
$$

The corresponding synthesis filter is $F_{A, i}(\omega)=Q_{A, i}^{*}(\omega)+$ $Q_{A, i}^{\prime *}(\omega)$. Similarly, in Fig. 16(b) and (c), $H_{B, i}(\omega)$ consists of $Q_{B, i}(\boldsymbol{\omega})$ and $Q_{B, i}^{\prime}(\boldsymbol{\omega})$, and $H_{C, i}(\boldsymbol{\omega})$ consists of $Q_{C, i}(\boldsymbol{\omega})$ and $Q_{C, i}^{\prime}(\boldsymbol{\omega})$. (The subscripts $A, B$, and $C$ of the analysis and synthesis filters are only temporary and meant to distinguish the three cases in Fig. 16.) Each analysis filter consists of two parallelograms. Therefore, the CMFB constructed this way is a subclass of two-parallelogram filter banks. We observe that all 


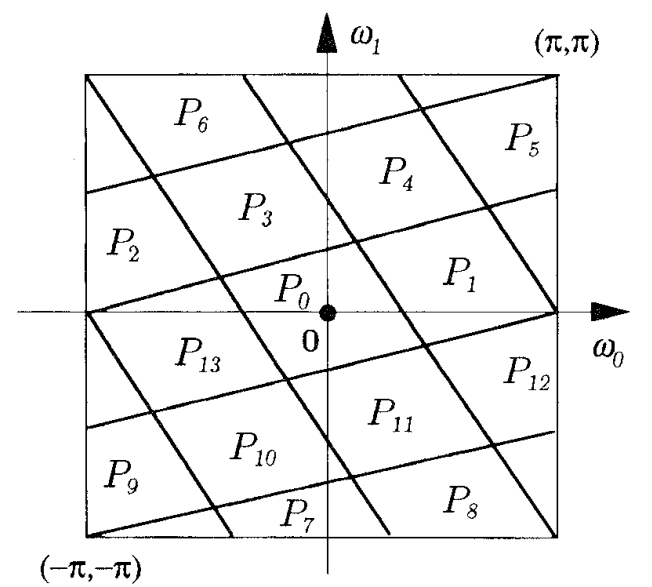

Fig. 14. DFT filter bank with decimation matrix $N$.

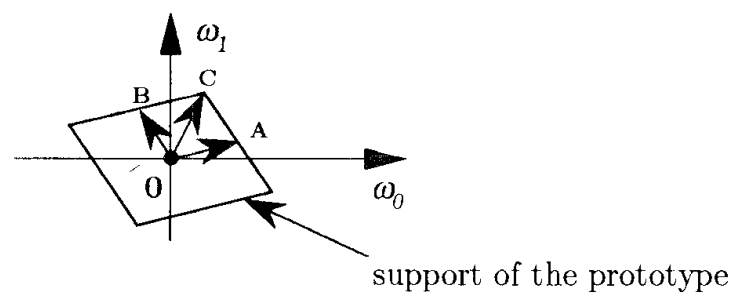

Fig. 15. Three possible vector shifts.

three support configurations are extension of the 1-D version. The three configurations will be referred to as configuration $A, B$, and $C$ in the discussion to follow. From Fig. 16, it seems that configurations $A$ and $B$ are very similar. Indeed, as we will see in issue 2 and 3 , properties derived for configurations $A$ are also true for configuration $B$, except for some minor modifications.

\section{Issue 2- $\mathcal{A} \mathcal{F} \mathcal{S}(M)$ Support Configuration}

We now study the conditions such that configurations $A, B$, and $C$ are $\mathcal{A} \mathcal{F} \mathcal{S}(\boldsymbol{M})$. As $M$ is fixed, and supports of the analysis filters in each configuration are already determined, Theorem 3.2 implies that $L$ will completely determine whether the the analysis filters are $\mathcal{A} \mathcal{F} \mathcal{S}(\boldsymbol{M})$ in the ideal case. In configuration $A$, the two passbands of the analysis filters are separated by $2 \pi N^{-T}\left(2 \boldsymbol{k}+\left[\begin{array}{ll}1 & 0\end{array}\right]^{T}\right)$ for some integer vector $\boldsymbol{k}$. By Theorem 3.2, the analysis filters are $\mathcal{A} \mathcal{F} \mathcal{S}(\boldsymbol{M})$ in ideal case in the following two situations. 1) Choose $L$ as in (b) of (1) for any $0<p \leq 1.2$ ) Choose $L$ as in (c) of (1) with $p=1$, which yields quincunx $L A T\left(\boldsymbol{L}^{T}\right)$ (see Fig. 12). Similarly, in configurations $B$ and $C$, the two passbands of the analysis filters are separated, respectively, by $2 \pi N^{-T}\left(2 k+\left[\begin{array}{ll}0 & 1\end{array}\right]^{T}\right)$ and $2 \pi \boldsymbol{N}^{-T}\left(2 \boldsymbol{k}+\left[\begin{array}{ll}1 & 1\end{array}\right]^{T}\right)$. We can verify that configuration $B$ is $\mathcal{A} \mathcal{F} \mathcal{S}(\boldsymbol{M})$ if we choose $\boldsymbol{L}$ as in (a) of (1) for any $0<p \leq 1$, or we can choose $L$ as in (c) of (1) with $p=1$, i.e., quincunx $\operatorname{LAT}\left(\boldsymbol{L}^{T}\right)$. Additionally, configuration $C$ is $\mathcal{A} \mathcal{F} \mathcal{S}(\boldsymbol{M})$ if we choose $L$ as in (a) or (b) of (1) for any $0<p \leq 1$.

Notice that in all three configurations, the analysis filters form a tiling of the frequency plane. When the configurations

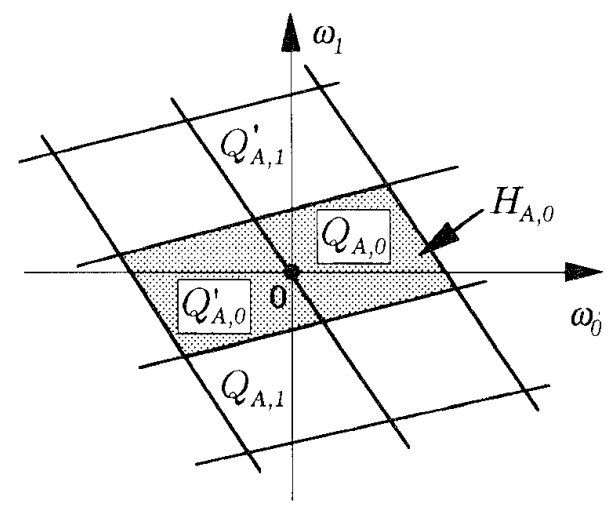

(a)

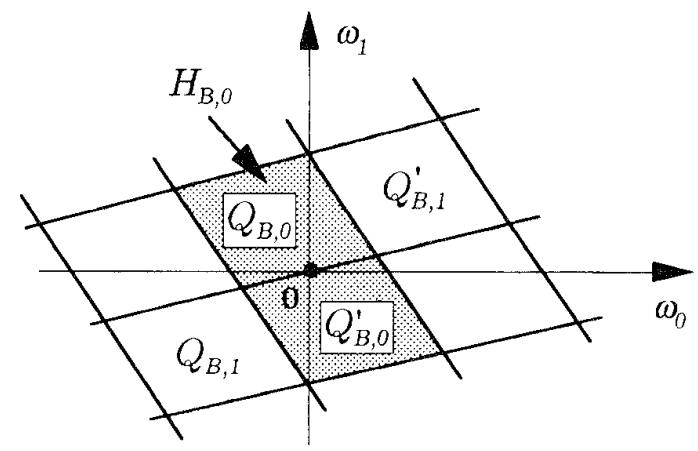

(b)

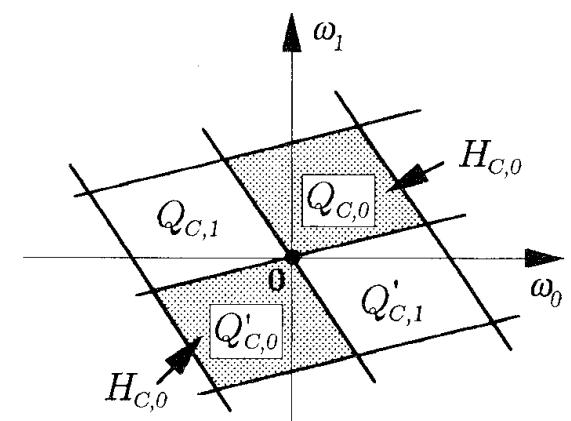

(c)

Fig. 16. Threc possible support configurations of the two-parallelogram cosine modulated filter bank. (a) Configuration $A$. (b) Configuration $B$. (c) Configuration $C$.

are $\mathcal{A} \mathcal{F} \mathcal{S}(\boldsymbol{M})$, the filter bank has perfect reconstruction if the prototype is an ideal filter.

\section{Issue 3-Support Permissibility}

According to the preceding analysis, each of the three support configurations is $\mathcal{A F \mathcal { S }}(\boldsymbol{M})$ for some $\boldsymbol{L}$. However, not all configurations are valid candidates in terms of support permissibility. From the discussion of two-parallelogram filters in Section IV, we know that when $\boldsymbol{L}^{T}$ is as in (a) or (b) of (1), one passband is edge adjacent to its own images, which leads to edge nonpermissibility. The 2-D CMFB studied in [14] usually has this type of nonpermissible support. From the consideration of support permissibility, any configuration with $L$ as in (a) or (b) of (1) is not suitable for the development of 
TABLE I

Sampling Criterion, Edge Permissibllity and Relation to the. Various Cases of $L$ in Eq. (1) for Configurations $A, B$ and $C$

\begin{tabular}{c|c|c|c} 
L & $\begin{array}{c}\text { (a) of Eq. (1) } \\
0<\mathrm{p} \leq 1\end{array}$ & $\begin{array}{c}\text { (b) of Eq. (1) } \\
0<\mathrm{p} \leq 1\end{array}$ & $\begin{array}{c}\text { (c) of Eq. (1) } \\
\mathrm{p=1} \text { (quincunx } L A T\left(\mathrm{~L}^{T}\right) \text { ) }\end{array}$ \\
\hline A & $\square$ & $\square$ \\
\hline B & $\square$ & $\square$ & $\square \square$ \\
\hline
\end{tabular}

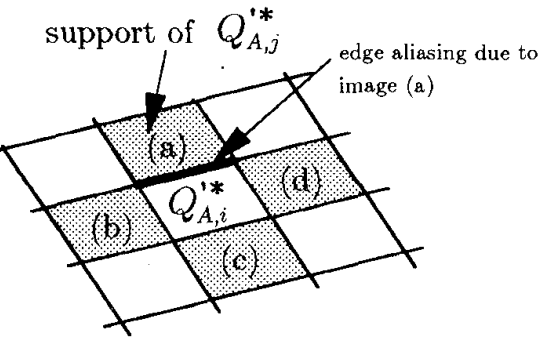

Images of $Q_{A, i}$

Fig. 17. Images of $Q_{\Lambda, i}(\omega)$ and their positions relative to $Q_{\Lambda, i}^{*}(\omega)$; four images of $Q_{A, i}(\omega)$ are edge-adjacent to $Q_{A, i}^{*}(\omega)$.

2-D CMFB. As configuration $C$ is $\mathcal{A} \mathcal{F S}(\boldsymbol{M})$ only for these two types of $\boldsymbol{L}$, configuration $C$ will not be considered. In addition, configurations $A$ and $B$ with $L$ as in (a) and (b) of (1) will not be considered. To design a edge permissible two-parallelogram CMFB, the only two possible choices left are configuration $A$ and $B$ with quincunx $\operatorname{LAT}\left(\boldsymbol{L}^{T}\right)$. Indeed, we will explain below that these two choices lead to edge permissible two-parallelogram CMFB.

As we discussed in Section IV, for two-parallelogram filters, each of the two passbands and its images form a check pattern when $\operatorname{LAT}\left(\boldsymbol{L}^{T}\right)$ is quincunx (Fig. 11(e)). Therefore, each passband is edge adjacent to four images of the other passband, which results in edge aliasing (Fig. 17). However, we can show that these edge aliasings appear in pairs, and the two-parallelogram CMFB is edge permissible. To see this, consider the $i$ th subband in configuration $A$ and only image (a), which is at the support of $Q_{A, j}^{\prime}(\omega)$. The resulting edge aliasing is as shown in Fig. 17. On the other hand, four images of $Q_{A, j}(\boldsymbol{\omega})$ will be edge adjacent to $Q_{A, j}^{\prime}$; one of the four images is at $Q_{A, i}^{\prime}$ and results in edge aliasing. It turns out that these two edge aliasing errors contribute to the same alias transfer function. Therefore, edge aliasing errors appear in pairs, and configuration $A$ is edge permissible. The situation for configuration $B$ is similar. In Section VI, we will discuss in detail how those edge aliasing errors can cancel one another.

Although configurations $A$ and $B$ can be edge permissible, they are not vertex permissible. For each analysis filter, images of one passband will be vertex adjacent to itself. The analysis in previous section shows that the resulting vertex aliasing is uncancelable under the assumption of good analysis and synthesis filters. Table $I$ is a summary of the role of $L$ to the $\mathcal{A} \mathcal{F} \mathcal{S}(\boldsymbol{M})$ properly and permissibility of each configuration. We see that configuration $C$ is either violating sampling criterion or edge nonpermissible. Configurations $A$ or $B$ can satisfy the sampling criterion and, at the same time, is edge permissible if $L A T\left(\boldsymbol{L}^{T}\right)$ is quincunx (Fig. 12). These two cases are more suitable for the construction of two-parallelogram CMFB. In this case, the two-parallelogram CMFB can have edge-based permissibility but lacks vertexbased permissibility. This imposes limitations on the attenuation of the individual filters in the two-parallelogram CMFB.

\section{FORMULATION OF THE TwO-PARALlELOGRAM CMFB'S}

We have set up the framework of two-parallelogram CMFB in Section IV. We have also derived two edge permissible support configurations. In this section, we will consider all the design details. For a given 2-D filter bank with decimation matrix $\boldsymbol{M}$, we start from a uniform DFT filter bank with twice the number of channels. By shifting the DFT filters property, we obtain two edge permissible configurations. We then proceed to formulate the analysis and synthesis filters.

\section{A. Configuration $A$ and $B$}

Consider the $|\boldsymbol{M}|$-channel 2-D filter bank with decimation matrix $M$ in Fig. 1. We start from a uniform DFT filter bank with decimation matrix $N=M L$, where $L$ is an integer matrix with $|\boldsymbol{L}|=2$ and quincunx $\operatorname{LAT}\left(\boldsymbol{L}^{T}\right)$ (for edge permissibility). To be more specific about the formulation of the DFT filter bank, let $M$ be diagonalized as $M=U \Lambda_{M} V_{M}$, where $U$ and $V_{M}$ are unimodular. The matrix $\Lambda_{M}$ is diagonal with diagonal elements $\left[\boldsymbol{\Lambda}_{\boldsymbol{M}}\right]_{00}=\lambda_{0}>0$ and $\left[\boldsymbol{\Lambda}_{\boldsymbol{M}}\right]_{11}=$ $\lambda_{1}>0$. For simplicity, we choose $L=\boldsymbol{V}_{\boldsymbol{M}}^{-1}\left[\begin{array}{ll}1 & 0 \\ 0 & 2\end{array}\right] \boldsymbol{V}$ for some unimodular $\boldsymbol{V}$ such that $L A T\left(\boldsymbol{L}^{T}\right)$ is quincunx. Then, the matrix $N$ given by $N=M L$ becomes

$$
\begin{aligned}
& \boldsymbol{N}=\underbrace{\boldsymbol{U}\left[\begin{array}{cc}
\lambda_{0} & 0 \\
0 & \lambda_{1}
\end{array}\right] \boldsymbol{V}_{\boldsymbol{M}}}_{\boldsymbol{M}} \underbrace{\boldsymbol{V}_{\boldsymbol{M}}^{-1}\left[\begin{array}{ll}
1 & 0 \\
0 & 2
\end{array}\right] \boldsymbol{V}}_{\boldsymbol{L}} \text { or } \\
& \boldsymbol{N}=\boldsymbol{U} \underbrace{\left[\begin{array}{cc}
\lambda_{\mathbf{0}} & 0 \\
0 & 2 \lambda_{1}
\end{array}\right]}_{\mathbf{\Lambda}} \boldsymbol{V}
\end{aligned}
$$

Clearly, $|\boldsymbol{M}|=\lambda_{0} \lambda_{1},|\boldsymbol{L}|=2$, and $|\boldsymbol{N}|=2 \lambda_{0} \lambda_{1}$. The prototype filter $P(\omega)$ in the DFT filter bank has a parallelogram support $S P D\left(\pi \boldsymbol{N}^{-T}\right)$. The DFT filters are shifted versions of the prototype by $2 \pi \boldsymbol{N}^{-T} \boldsymbol{k}_{i}$, for $\boldsymbol{k}_{i} \in \mathcal{N}\left(\boldsymbol{N}^{T}\right)$.

For referring convenience, we will adopt a particular ordering of $\mathcal{N}\left(\boldsymbol{N}^{T}\right)$. As $\boldsymbol{V}^{T} \boldsymbol{\Lambda}$ and $\boldsymbol{N}^{T}$ have the same lattices, 
we will consider the ordering of $\mathcal{N}\left(\boldsymbol{V}^{T} \boldsymbol{\Lambda}\right)$ for simplicity. The ordering is as follows:

$$
\begin{gathered}
\boldsymbol{k}_{k_{0}+\lambda_{0} k_{1}}=\boldsymbol{V}^{T}\left[\begin{array}{l}
k_{0} \\
k_{1}
\end{array}\right], \quad k_{0}=0,1, \cdots, \lambda_{0}-1, \\
k_{1}=0,1, \cdots, 2 \lambda_{1}-1 .
\end{gathered}
$$

Then, $\mathcal{N}\left(\boldsymbol{V}^{T} \boldsymbol{\Lambda}\right)=\left\{\boldsymbol{k}_{0}, \boldsymbol{k}_{1}, \cdots, \boldsymbol{k}_{\mid \boldsymbol{N}_{-1}}\right\}$. Configurations $A$ and $B$ (Fig. 16) are obtained by shifting the DFT filters. In particular, the complex filter underlying configurations $A$ and $B$ are

$$
Q_{\boldsymbol{k}}(\boldsymbol{\omega})=P\left(\boldsymbol{\omega}-2 \pi \boldsymbol{N}^{-T}(\boldsymbol{k}+\boldsymbol{l})\right), \quad \boldsymbol{k} \in \mathcal{N}\left(\boldsymbol{V}^{T} \boldsymbol{\Lambda}\right)
$$

where $\boldsymbol{l}=\left[\begin{array}{ll}0.5 & 0\end{array}\right]^{T}$ for configuration $A$ and $\boldsymbol{l}=\left[\begin{array}{ll}0 & 0.5\end{array}\right]^{T}$ for configuration $B$. The vector subscript of $Q_{\boldsymbol{k}}(\omega)$ should be interpreted modulo $N^{T}$.

\section{B. The Analysis and Synthesis Filters}

To obtain real-coefficient analysis and synthesis filters, we need to combine two shifted copies of the prototype $P(\boldsymbol{\omega})$. Suppose the impulse response of $Q_{\boldsymbol{k}}(\boldsymbol{\omega})$ is $q_{\boldsymbol{k}}(\boldsymbol{n})$. Let $k^{\prime}$ be such that $q_{\boldsymbol{k}^{\prime}}(\boldsymbol{n})=q_{\boldsymbol{k}}^{*}(\boldsymbol{n})$. Pair $Q_{\boldsymbol{k}}(\boldsymbol{\omega})$ and $Q_{\boldsymbol{k}^{\prime}}(\boldsymbol{\omega})$. Then, the analysis filters of the form $Q_{\boldsymbol{k}}(\boldsymbol{\omega})+Q_{\boldsymbol{k}}(\boldsymbol{\omega})$ have real coefficients. The pairing procedure is formulated in the following property.

Proposition 5.1: The coefficients of $Q_{\boldsymbol{k}_{n_{0}+m_{1} \lambda_{0}}}(\boldsymbol{\omega})$ and $Q_{\boldsymbol{k}_{m_{0}^{\prime}+m_{1}^{\prime} \lambda_{0}}}(\boldsymbol{\omega})$ are complex conjugates of each other if $m_{0}, m_{0}^{\prime}, m_{1}$, and $m_{1}^{\prime}$ are related by

$$
\begin{aligned}
& m_{0}+m_{0}^{\prime}=b_{0} \bmod \lambda_{0}, \quad \text { and } \\
& m_{1}+m_{1}^{\prime}=b_{1} \bmod 2 \lambda_{1}, \quad \text { where } \quad\left[\begin{array}{l}
b_{0} \\
b_{1}
\end{array}\right]=-2 \boldsymbol{V}^{-T} \boldsymbol{l} .
\end{aligned}
$$

Proof of Proposition 5.1: The filter $Q_{\boldsymbol{k}}(\omega)$ is a shift of the prototype $P(\boldsymbol{\omega})$ by $2 \pi \boldsymbol{N}^{-T}(\boldsymbol{k}+\boldsymbol{L})$. If $Q_{\boldsymbol{k}^{\prime}}(\boldsymbol{\omega})$ is a shift of the prototype $P(\boldsymbol{\omega})$ by $-2 \pi \boldsymbol{N}^{-T}(\boldsymbol{k}+\boldsymbol{l}), q_{\boldsymbol{k}}(\boldsymbol{n})$ and $q_{\boldsymbol{k}^{\prime}}(\boldsymbol{n})$ are conjugates of each other. Therefore, $Q_{\boldsymbol{k}}(\boldsymbol{\omega})+Q_{\boldsymbol{k}^{\prime}}(\boldsymbol{\omega})$ has real coefficients if $2 \pi \boldsymbol{N}^{-T}\left(\boldsymbol{k}^{\prime}+\boldsymbol{l}\right)=-2 \pi \boldsymbol{N}^{-T}(\boldsymbol{k}+\boldsymbol{l}) \bmod 2 \pi \boldsymbol{I}$, or equivalently,

$$
\boldsymbol{k}+\boldsymbol{k}^{\prime}=-2 \boldsymbol{l} \bmod N^{T}
$$

Next, we would like to relate $m$ and $m^{\prime}$ such that $\boldsymbol{k}_{m}+\boldsymbol{k}_{m^{\prime}}=$ $-2 \boldsymbol{l} \bmod \boldsymbol{N}^{T}$. Let $m=m_{0}+\lambda_{0} m_{1}$ and $m^{\prime}=m_{0}^{\prime}+\lambda_{0} m_{1}^{\prime}$. Then,

$$
\begin{aligned}
& \boldsymbol{k}_{m}=\boldsymbol{V}^{T}\left[\begin{array}{l}
m_{0} \\
m_{1}
\end{array}\right], \\
& \boldsymbol{k}_{m^{\prime}}=\boldsymbol{V}^{T}\left[\begin{array}{l}
m_{0}^{\prime} \\
m_{1}^{\prime}
\end{array}\right] \text { and therefore } \\
& \boldsymbol{V}^{T}\left[\begin{array}{l}
m_{0}+m_{0}^{\prime} \\
m_{1}+m_{1}^{\prime}
\end{array}\right] \boldsymbol{N}^{\Upsilon}-2 \boldsymbol{l} .
\end{aligned}
$$

It follows that $Q_{\boldsymbol{k}_{m_{0}+m_{1} \lambda_{0}}}(\boldsymbol{\omega})+Q_{\boldsymbol{k}_{m n_{0}^{\prime}+m_{1}^{\prime} \lambda_{0}}}(\boldsymbol{\omega})$ have real coefficients if $m_{0}, m_{0}^{\prime}, m_{1}$, and $m_{1}^{\prime}$ are related as in (7).
If we combine conjugate pairs $Q_{\boldsymbol{k}}(\boldsymbol{\omega})$ and $Q_{\boldsymbol{k}^{\prime}}(\boldsymbol{\omega})$ as described in Proposition 5.1, we get $|\boldsymbol{M}|$ real-coefficient analysis filters.

$$
\begin{aligned}
H_{m}(\boldsymbol{\omega}) & =Q_{\boldsymbol{k}_{m_{0}+m_{1} \lambda_{0}}}(\boldsymbol{\omega})+Q_{\boldsymbol{k}_{m_{0}^{\prime}+m_{1}^{\prime} \lambda_{0}}}(\boldsymbol{\omega}), \\
F_{m}(\boldsymbol{\omega}) & =H_{m}^{*}(\boldsymbol{\omega}), \quad m=0,1, \cdots,|\boldsymbol{M}|-1
\end{aligned}
$$

where $m_{0}=m \bmod \lambda_{0}, m_{1}=b+\left(m-m_{0}\right) / \lambda_{0} \bmod 2 \lambda_{1}$, and $b=\left(b_{1}+1\right) / 2$. Values of $m_{0}^{\prime}$ and $m_{1}^{\prime}$ are given by (7). It can be verified that $b=\left(b_{1}+1\right) / 2$ is an integer, and the above enumeration of the analysis filters is complete. It follows from (9) that the impulse response of the analysis filter $H_{m}(\boldsymbol{\omega})$ is

$$
\begin{gathered}
h_{m}(\boldsymbol{n})=2 p(\boldsymbol{n}) \cos \left(2 \pi\left(\boldsymbol{k}_{m_{0}+m_{1} \lambda_{0}}+\boldsymbol{l}\right)^{T} \boldsymbol{N}^{-1} \boldsymbol{n}\right), \\
m=0,1, \cdots,|\boldsymbol{M}|-1 .
\end{gathered}
$$

We have chosen $F_{m}(\boldsymbol{\omega})=H_{m}^{*}(\boldsymbol{\omega})$ in the above formulation, as the system is eventually going to be paraunitary. The impulse responses of the analysis and synthesis filters are related by

$$
f_{k}(\boldsymbol{n})=h_{k}(\boldsymbol{- n}), \quad \text { where } \boldsymbol{n} \text { is any } 2 \times 1 \text { vector. }
$$

In a 1-D CMFB, each analysis filter has two distinct shifts of the prototype filter. Therefore, the total bandwidth of each individual filter is the same. For 2-D filters, total bandwidth should be interpreted as the total spectral occupancy. In the two-parallelogram CMFB, all analysis filters have the same size of spectral occupancy. Every analysis filter consists of two distinct shifts of the prototype. This follows from the quincunx property of $L A T\left(\boldsymbol{L}^{T}\right)$ as we will show below.

Proposition 5.2: The analysis and synthesis filters have equal size of spectral occupancy.

Proof of Proposition 5.2: We only need to show that $Q_{\boldsymbol{k}}(\boldsymbol{\omega})$ and $Q_{\boldsymbol{k}^{\prime}}(\boldsymbol{\omega})$ are distinct filters whenever the coefficients of $Q_{\boldsymbol{k}}(\boldsymbol{\omega})$ and $Q_{\boldsymbol{k}^{\prime}}(\boldsymbol{\omega})$ are complex conjugate of each other. Suppose the contrary is true; the coefficients of $Q_{k}(\omega)$ and $Q_{\boldsymbol{k}^{\prime}}(\boldsymbol{\omega})$ are complex conjugate of each other, but they are the same filter, i.e., $k=k^{\prime} \bmod N^{T}$. By (8), we have

$$
2 \boldsymbol{k}^{\stackrel{N^{T}}{=}-2 \boldsymbol{l}} \text {. }
$$

As $L A T\left(\boldsymbol{L}^{T}\right)$ is quincunx, $2 \boldsymbol{k}=\mathbf{0} \bmod L^{T}$. Taking modulo $L^{T}$ on both sides of the above equation, we have $0 \stackrel{L^{T}}{=}-2 \boldsymbol{l}$. By the definition of $\boldsymbol{l}, 2 \boldsymbol{l}=\left[\begin{array}{ll}1 & 0\end{array}\right]^{T}$ or $\left[\begin{array}{ll}0 & 1\end{array}\right]^{T}$. Therefore, $2 \boldsymbol{l} \notin L A T\left(\boldsymbol{L}^{T}\right)$, which leads to a contradiction. We conclude that when the coefficients of $Q_{\boldsymbol{k}}(\omega)$ and $Q_{\boldsymbol{k}^{\prime}}(\boldsymbol{\omega})$ are complex conjugate of each other, $Q_{\boldsymbol{k}}(\omega)$ and $Q_{\boldsymbol{k}^{\prime}}(\omega)$ are not the same, and each analysis filter consists of two distinct copies of the prototype.

\section{PERFECT ReCONSTRUCTION Two-PARALLELOGRAM CMFB'S}

In this section, we first show how to cancel edge aliasing errors that arise in every subband. Then, we present the necessary and sufficient condition for perfect reconstruction of a two-parallelogram CMFB. In the 1-D case, the CMFB has perfect reconstruction if and only if the polyphase components of the prototype are pairwise power complementary [28]. We will see that similar necessary and sufficient conditions can be derived for two-parallelogram CMFB as well. 


\section{A. Cancelation of Edge Aliasing}

In Section IV, we mentioned that in each subband (configuration $A$ or $B$ ), one passband of each analysis filter is edge adjacent to four images of the other passband. This results in serious edge aliasing. It turns out that these edge aliasing errors from different subband can actually cancel with one another if the prototype has linear phase and satisfies some minor condition. To be more specific, let the impulse response of the prototype be $p(\boldsymbol{n})$ and

$$
p(\boldsymbol{n})=p\left(\boldsymbol{n}_{s}-\boldsymbol{n}\right), \quad \text { for some integer vector } \boldsymbol{n}_{s} .
$$

Proposition 6.1: Consider a two-parallelogram CMFB with analysis and synthesis given as in (9). Let $L A T\left(\boldsymbol{L}^{T}\right)$ be quincunx. Then, the following is true:

1) Edge aliasing errors appear in pairs.

2) Pairwise edge aliasings can cancel with each other if $\boldsymbol{n}_{s}$ satisfies

$$
\boldsymbol{n}_{s}=\boldsymbol{N}\left[\begin{array}{l}
0.5 \\
0.5
\end{array}\right] \bmod N .
$$

3) The vector $\boldsymbol{n}_{s}$ thus determined is an integer vector, and $\boldsymbol{n}_{s}$ has the form $\boldsymbol{n}_{s} \stackrel{N}{=} \boldsymbol{U}\left[\begin{array}{ll}0 & \lambda_{1}\end{array}\right]^{T}$.

This is a minor condition because it is always possible to shift the linear-phase prototype such that $(11)$ is satisfied.

Proof of Proposition 6.1:

1) Consider the subband that has analysis filter $Q_{\boldsymbol{k}}(\boldsymbol{\omega})+$ $Q_{\boldsymbol{k}^{\prime}}(\boldsymbol{\omega})$. The aliasing terms due to edge adjacency of $Q_{\boldsymbol{k}^{\prime}}^{*}(\boldsymbol{\omega})$, and images of $Q_{\boldsymbol{k}}(\boldsymbol{\omega})$ are

$$
Q_{\boldsymbol{k}^{\prime}+\boldsymbol{k}_{s}}(\boldsymbol{\omega}) Q_{\boldsymbol{k}^{\prime}}^{*}(\boldsymbol{\omega}), \quad \text { where } \boldsymbol{k}_{\mathrm{s}} \in \mathrm{AS} .
$$

The adjacency set (AS) is given by

$$
\mathrm{AS} \triangleq\left\{\left[\begin{array}{l}
1 \\
0
\end{array}\right],\left[\begin{array}{r}
-1 \\
0
\end{array}\right],\left[\begin{array}{l}
0 \\
1
\end{array}\right],\left[\begin{array}{r}
0 \\
-1
\end{array}\right]\right\} .
$$

These aliasing terms contribute, respectively, to the aliasing transfer function $A_{\boldsymbol{L}^{-T}\left({ }_{2} \boldsymbol{k}^{\prime}+2 \boldsymbol{l}+\boldsymbol{k}_{s}\right)}(\boldsymbol{\omega})$. Now, consider the subband that has analysis filter $Q_{\boldsymbol{k}-\boldsymbol{k}}(\boldsymbol{\omega})+$ $Q_{\boldsymbol{k}^{\prime}+\boldsymbol{k}_{s}}(\boldsymbol{\omega})$. One image of $Q_{\boldsymbol{k}-\boldsymbol{k}_{s}}(\boldsymbol{\omega})$ will be located in support of $Q_{\boldsymbol{k}^{\prime}}(\boldsymbol{\omega})$ and results in aliasing error

$$
Q_{\boldsymbol{k}^{\prime}+\boldsymbol{k}_{s}}^{*}(\boldsymbol{\omega}) Q_{\boldsymbol{k}^{\prime}}(\boldsymbol{\omega})
$$

which also contributes to $A_{\boldsymbol{L}^{-T}\left(2 \boldsymbol{k}^{\prime}+2 \boldsymbol{l}+\boldsymbol{k}_{s}\right)}(\boldsymbol{\omega})$. Therefore, the edge aliasing errors occur in pairs.

2) Notice that the error in (13) is the conjugate of error in (12), and they cancel each other if

$Q_{\boldsymbol{k}^{\prime}}^{*}(\boldsymbol{\omega}) Q_{\boldsymbol{k}^{\prime}+\boldsymbol{k}_{s}}(\boldsymbol{\omega})=-Q_{\boldsymbol{k}^{\prime}}(\boldsymbol{\omega}) Q_{\boldsymbol{k}^{\prime}+\boldsymbol{k}_{s}}^{*}(\boldsymbol{\omega}), \quad \forall \boldsymbol{k}_{i} \in \mathrm{AS}$.

In what follows, we show that the above equation holds when $\boldsymbol{n}_{s}$ is as in (11). By (10), $P(\boldsymbol{\omega})$ assumes the form $P(\boldsymbol{\omega})=e^{-j \boldsymbol{\omega}^{T} \boldsymbol{n}_{s} / 2} P_{r}(\boldsymbol{\omega})$, where $P_{r}(\boldsymbol{\omega})$ is a real-valued function. Let $P_{r, \boldsymbol{k}}(\boldsymbol{\omega})=P_{r}\left(\boldsymbol{\omega}-2 \pi \boldsymbol{N}^{-T}(\boldsymbol{k}+\boldsymbol{l})\right)$. Then, we can verify

$$
\begin{aligned}
& Q_{\boldsymbol{k}^{\prime}}^{*}(\boldsymbol{\omega}) Q_{\boldsymbol{k}^{\prime}+\boldsymbol{k}_{s}}(\boldsymbol{\omega})+Q_{\boldsymbol{k}^{\prime}}(\boldsymbol{\omega}) Q_{\boldsymbol{k}^{\prime}+\boldsymbol{k}_{\mathrm{s}}}^{*}(\boldsymbol{\omega}) \\
& =\left(e^{j \pi \boldsymbol{k}_{s}^{T} \boldsymbol{N}^{-1} \boldsymbol{n}_{s}}+e^{-j \pi \boldsymbol{k}_{s}^{T} \boldsymbol{N}^{-1} \boldsymbol{n}_{s}}\right) P_{r, \boldsymbol{k}^{\prime}}(\boldsymbol{\omega}) \\
& \cdot P_{r, \boldsymbol{k}^{\prime}+\boldsymbol{k}_{s}}(\boldsymbol{\omega})
\end{aligned}
$$

In view of the above equation, the condition for alias cancelation is $e^{j 2 \pi \boldsymbol{k}_{s}^{T} \boldsymbol{N}^{-1} \boldsymbol{n}_{s}}=-1, \forall \boldsymbol{k}_{s} \in \mathrm{AS}$. Since $e^{j 2 \pi \boldsymbol{k}_{s}^{T} \boldsymbol{N}^{-1} \boldsymbol{n}_{s}}=e^{-j 2 \pi \boldsymbol{k}_{s}^{T} \boldsymbol{N}^{-1} \boldsymbol{n}_{s}}$, it is necessary and sufficient that $e^{j 2 \pi \boldsymbol{k}_{s}^{T} \boldsymbol{N}^{-1} \boldsymbol{n}_{s}}=-1$ for $\boldsymbol{k}_{s}=\left[\begin{array}{ll}1 & 0\end{array}\right]^{T}$ and $\boldsymbol{k}_{\mathrm{s}}=\left[\begin{array}{ll}0 & 1\end{array}\right]^{T}$. That is,

$$
\begin{aligned}
2 \pi\left[\begin{array}{ll}
1 & 0 \\
0 & 1
\end{array}\right] \boldsymbol{N}^{-1} n_{s} & \\
& =2 \pi \boldsymbol{d}+\pi\left[\begin{array}{l}
1 \\
1
\end{array}\right], \text { for some integer vector } \boldsymbol{d}
\end{aligned}
$$

Premultiplying both sides by $(1 / 2 \pi) N$, we have $\boldsymbol{n}_{s}=\boldsymbol{N} \boldsymbol{d}+\boldsymbol{N}\left[\begin{array}{ll}0.5 & 0.5\end{array}\right]^{T}$ or, equivalently, $\boldsymbol{n}_{s}=$ $N\left[\begin{array}{ll}0.5 & 0.5\end{array}\right]^{T} \bmod N$.

3) The vector $\boldsymbol{n}_{s}$ is determined by $\boldsymbol{n}_{s}=\boldsymbol{U} \boldsymbol{\Lambda} \boldsymbol{V}\left[\begin{array}{ll}0.5 & 0.5\end{array}\right]^{T}$ $\bmod \boldsymbol{N}$. As $\boldsymbol{L}^{T}=\boldsymbol{V}^{T}\left[\begin{array}{ll}1 & 0 \\ 0 & 2\end{array}\right] \boldsymbol{V}_{\boldsymbol{M}}^{-T}, L A T\left(\boldsymbol{L}^{T}\right)$ is the same as the lattice of $\boldsymbol{V}^{T}\left[\begin{array}{ll}1 & 2 \\ 0 & 2\end{array}\right]$. Let

$\boldsymbol{V}=\left[\begin{array}{ll}v_{00} & v_{01} \\ v_{10} & v_{11}\end{array}\right], \quad$ then $\boldsymbol{V}^{T}\left[\begin{array}{ll}1 & 0 \\ 0 & 2\end{array}\right]=\left[\begin{array}{ll}v_{00} & 2 v_{10} \\ v_{01} & 2 v_{11}\end{array}\right]$.

The vector $\left[\begin{array}{ll}v_{00} & v_{01}\end{array}\right]^{T}$ is on the lattice of $\boldsymbol{L}^{T}$, which is quincunx. Any vector $c$ on the quincunx lattice has the property that $[\boldsymbol{c}]_{0}+[\boldsymbol{c}]_{1}$ is even. This means that $v_{00}+v_{01}$ is even; $v_{00}$ and $v_{01}$ are either both odd or both even. If $v_{00}$ and $v_{01}$ are both even, then $|\boldsymbol{V}|$ is even, which contradicts with the assumption that $V$ is unimodular and has $|\boldsymbol{V}|=1$. Therefore, $v_{00}$ and $v_{01}$ are both odd. This in turn implies that $v_{10}$ and $v_{11}$ must be one odd and one even (since $|\boldsymbol{V}|=1$ ). As a result $V\left[\begin{array}{ll}0.5 & 0.5\end{array}\right]^{T}=\left[\begin{array}{ll}c_{0} & c_{1}+0.5\end{array}\right]^{T}$ for some integers $c_{0}$ and $c_{1}$. Let $\boldsymbol{c}=\left[\begin{array}{ll}c_{0} & c_{1}\end{array}\right]^{T}$. Then, $\boldsymbol{n}_{s}=\boldsymbol{U} \boldsymbol{\Lambda}\left(\boldsymbol{c}+\left[\begin{array}{ll}0 & 0.5\end{array}\right]^{T}\right)$ or, equivalently, $\boldsymbol{n}_{s}=\boldsymbol{N}\left(\boldsymbol{V}^{-1} \boldsymbol{c}\right)+\boldsymbol{U}\left[\begin{array}{ll}0 & \lambda_{1}\end{array}\right]^{T}$. Hence, we have $\boldsymbol{n}_{s} \stackrel{N}{=} \boldsymbol{U}\left[\begin{array}{ll}0 & \lambda_{1}\end{array}\right]^{T}$, which is an integer vector.

\section{B. Perfect Reconstruction Conditions}

Let the prototype have the following polyphase representation:

$$
P(\boldsymbol{\omega})=\sum_{i=0}^{|\boldsymbol{N}|-1} E_{i}\left(\boldsymbol{N}^{T} \boldsymbol{\omega}\right) e^{-j \boldsymbol{\omega}^{T} \boldsymbol{n}_{i}}
$$

where

$$
\begin{gathered}
\boldsymbol{n}_{n_{0}+\lambda_{0} n_{1}}=\boldsymbol{U}\left[\begin{array}{l}
n_{0} \\
n_{1}
\end{array}\right], \quad n_{0}=0,1, \cdots, \lambda_{0}-1, \\
n_{1}=0,1, \cdots, 2 \lambda_{1}-1
\end{gathered}
$$

where $U$ is as given in (4). Then, $E_{i}(\boldsymbol{\omega})$ is the polyphase component of $P(\boldsymbol{\omega})$ with respect to $\boldsymbol{n}_{i}$. The paraunitariness of the two-parallelogram CMFB can be translated into pairwise power complementary conditions on $E_{i}(\boldsymbol{\omega})$.

Theorem 6.I-Necessary and Sufficient Conditions for Paraunitariness: Consider the filter bank with decimation matrix $M$ in Fig. 1 and the choice of analysis and synthesis filters in (9). Let the matrix $N$ be given by $N=M L$, where $L A T\left(L^{T}\right)$ is quincunx. In addition, let the prototype be linear-phase with $p(\boldsymbol{n})=p\left(\boldsymbol{n}_{s}-\boldsymbol{n}\right)$ and $\boldsymbol{n}_{s}=\boldsymbol{N}\left[\begin{array}{ll}0.5 & 0.5\end{array}\right]^{T} \bmod \boldsymbol{N}$. Then, the 
two-parallelogram CMFB is paraunitary (i.e., the polyphase matrix is paraunitary) if and only if

$$
E_{i}^{*}(\boldsymbol{\omega}) E_{i}(\boldsymbol{\omega})+E_{i+\lambda_{0} \lambda_{1}}^{*}(\boldsymbol{\omega}) E_{i+\lambda_{0} \lambda_{1}}(\boldsymbol{\omega})=c
$$

where $c$ is some constant.

The proof of Theorem 6.1 is given in Appendix B.

Remark on Theorem 6.1: The condition in (16) is equivalent to saying that $E_{i}(\boldsymbol{\omega})$ and $E_{i+\lambda_{0} \lambda_{1}}(\boldsymbol{\omega})$ are power complementary in a 2-D sense. As explained in Section $V$, the two-parallelogram CMFB is of configuration $A$ when $l=$ $\left[\begin{array}{ll}0.5 & 0\end{array}\right]^{T}$ and configuration $B$ when $l=\left[\begin{array}{ll}0 & 0.5\end{array}\right]^{T}$. As the theorem is true regardless of choice of $l$, Theorem 6.1 holds for both configuration $A$ and configuration $B$.

\section{Polyphase Components of the Prototype}

In the 1-D CMFB, the polyphase components of the prototype are related in pairs because of linear phase constraint of the prototype. At the same time, there are also power complementary pairs due to paraunitariness. Furthermore, if half of the polyphase components are pairwise power complementary, the other half, due to linear phase, are automatically pairwise power complementary as derived in [28]. The situation is similar in the two-parallelogram CMFB, as we elaborate upon next.

According to (10), the impulse response of the prototype is $p(\boldsymbol{n})=p\left(\boldsymbol{n}_{s}-\boldsymbol{n}\right)$. Therefore, the vector $\boldsymbol{n}_{s}$ determines the pairwise relations of the polyphase components. More precisely, we will show that the polyphase component are pairwise related by

$$
\begin{gathered}
E_{n_{0}+\lambda_{0} n_{1}}(\boldsymbol{\omega})=e^{-j \boldsymbol{\omega}^{T}\left(\boldsymbol{d}-\boldsymbol{V}^{-1}\left(c_{0} c_{1}\right)^{T}\right)} E_{n_{0}^{\prime}+\lambda_{0} n_{1}^{\prime}}^{*}(\boldsymbol{\omega}) \\
n_{0}^{\prime}=-n_{0} \bmod \lambda_{0} \\
n_{1}^{\prime}=\lambda_{1}-n_{1} \bmod 2 \lambda_{1}
\end{gathered}
$$

where $\boldsymbol{d}$ is a vector determined by $\boldsymbol{n}_{s}$, and the two quantities $c_{0}$ and $c_{1}$ are given by

$$
c_{0}=\left\{\begin{array}{ll}
0, & \text { if } n_{0}=0, \\
1, & \text { otherwise, }
\end{array} \text { and } \quad c_{1}= \begin{cases}0, & \text { if } n_{1} \leq \lambda_{1}, \\
1, & \text { otherwise. }\end{cases}\right.
$$

Proof of (17): As $n_{s}=N\left[\begin{array}{ll}0.5 & 0.5\end{array}\right]^{T} \bmod \boldsymbol{N}, \boldsymbol{n}_{s}=\boldsymbol{N d}+$ $N\left[\begin{array}{ll}0.5 & 0.5\end{array}\right]^{T}$ for some integer vector $d$. Using the linear phase property of $P(\boldsymbol{\omega})$, we have $P(\boldsymbol{\omega})=e^{-j \boldsymbol{\omega}^{T} \boldsymbol{n}_{s}} P^{*}(\boldsymbol{\omega})$. Substituting in the polyphase representation of $P(\omega)$ gives us

$$
\begin{aligned}
\sum_{n_{0}=0}^{\lambda_{0}-1} & \sum_{n_{1}=0}^{2 \lambda_{1}-1} E_{n_{0}+\lambda_{0} n_{1}}\left(N^{T} \omega\right) e^{-j \boldsymbol{\omega}^{T} U\left(n_{0} n_{1}\right)^{T}} \\
= & \sum_{n_{0}^{\prime}=0}^{\lambda_{0}-1} \sum_{n_{1}^{\prime}=0}^{2 \lambda_{1}-1} E_{n_{0}^{\prime}+\lambda_{0} n_{1}^{\prime}}^{*}\left(N^{T} \omega\right) e^{-j \boldsymbol{\omega}^{T} N \boldsymbol{d}} \\
& \cdot e^{-j \boldsymbol{\omega}^{T} \boldsymbol{U}\left(-n_{0}^{\prime} \lambda_{\mathrm{I}}-n_{1}^{\prime}\right)^{T}} .
\end{aligned}
$$

The right-hand side of the above equation is equal to

$$
\begin{gathered}
\sum_{n_{0}^{\prime}=0}^{\lambda_{0}-1} \sum_{n_{1}^{\prime}=0}^{2 \lambda_{1}-1} E_{n_{0}^{\prime}+\lambda_{0} n_{1}^{\prime}}^{*}\left(\boldsymbol{N}^{T} \boldsymbol{\omega}\right) e^{-j \boldsymbol{\omega}^{T} \boldsymbol{N}\left(\boldsymbol{d}-\boldsymbol{V}^{-1}\left(c_{0} c_{1}\right)^{T}\right)} \\
\cdot e^{-j \boldsymbol{\omega}^{T} \boldsymbol{U}\left(c_{0} \lambda_{0}-n_{0}^{\prime} 2 c_{1} \lambda_{1}+\lambda_{1}-n_{1}^{\prime}\right)^{T}} .
\end{gathered}
$$

Therefore,

$$
E_{n_{0}+\lambda_{0} n_{1}}\left(\boldsymbol{N}^{T} \boldsymbol{\omega}\right)=E_{n_{0}^{\prime}+\lambda_{0} n_{1}^{\prime}}^{*}\left(\boldsymbol{N}^{T} \boldsymbol{\omega}\right) e^{-j \boldsymbol{\omega}^{T} \boldsymbol{N}\left(\boldsymbol{d}-\boldsymbol{V}^{-1}\left(c_{0} c_{1}\right)^{T}\right)}
$$

if $\left[\begin{array}{ll}n_{0} & n_{1}\end{array}\right]^{T}=\left[\begin{array}{ll}c_{0} \lambda_{0}-n_{0}^{\prime} & 2 c_{1} \lambda_{1}+\lambda_{1}-n_{1}^{\prime}\end{array}\right]^{T}$. This gives us the pairwise relations given in (17).

According to the statement of Theorem 6.1, we know the first and the second halves of the polyphase components are power complementary in pairs. Combining these two different pairwise relations, we observe that if the following pairs

$$
\begin{aligned}
& {\left[E_{n_{0}+\lambda_{0} n_{1}}(\boldsymbol{\omega}), E_{n_{0}+\left(n_{1}+\lambda_{1}\right) \lambda_{0}}(\boldsymbol{\omega})\right]} \\
& \quad \text { for } 0 \leq n_{0}<\left\lceil\lambda_{0} / 2\right\rceil \text { and } 0 \leq n_{1}<\left\lceil\left(\lambda_{1}+1\right) / 2\right\rceil,
\end{aligned}
$$

are power complementary, then the others will be pairwise power complementary due to the linear phase property of the prototype. Here, the ceiling function $\lceil x\rceil$ is defined as the smallest integer greater than $x$.

Comment on the Nyquist Property of the Analysis Filters: As in the 1-D case, we can define Nyquist filters. A filter $h(\boldsymbol{m})$ is called a Nyquist $(\boldsymbol{N})$ filter if one polyphase component of $h(\boldsymbol{m})$ is a delay. From the previous discussion, we observe that while $\left(E_{0}(\boldsymbol{\omega}), E_{\lambda_{0} \lambda_{1}}(\boldsymbol{\omega})\right)$ is a power complementary pair, these two polyphase components are also related because $P(\omega)$ has linear phase. As a result, $E_{0}(\boldsymbol{\omega})$ and $E_{\lambda_{0} \lambda_{1}}(\boldsymbol{\omega})$ are merely delays. Therefore, the prototype is a Nyquist $(N)$ filter. The analysis filters are cosine-modulated versions of the prototype. We conclude that the analysis filters are also Nyquist $(N)$ filters.

\section{IMPLEMENTATION AND DESIGN EXAMPLE OF TWO-PARALLELOGRAM CMFB}

\section{Efficient Implementation of the Two-Parallelogram CMFB}

Efficient implementation is one of the reasons that CMFB's attract a lot of attention. In the 1-D CMFB, the complexity of the analysis bank or the synthesis bank is that of the prototype filter plus a DCT matrix. The DCT matrices are known to be low-complexity matrices. We will show that there also exists efficient implementation for the two-parallelogram CMFB. The cost of the analysis bank or the synthesis bank is that of a prototype filter plus a matrix, which has elements resembling that of a nonseparable 2-D DCT matrix. Implementation of this DCT-like matrix can be decomposed into 1-D DCT matrices of smaller dimensions. Denote the complexity of a $\lambda$-point DCT by $C(\lambda)$. Then, the complexity of $C$ is roughly $4 \lambda_{1} C\left(\lambda_{0}\right)+\lambda_{0} C\left(2 \lambda_{1}\right)$, where $\lambda_{0}$ and $\lambda_{1}$ are the diagonal elements of $\boldsymbol{A}_{\boldsymbol{M}}$.

Using the polyphase representation of the prototype in (14), the analysis filters in (9) can be rewritten as

$$
\begin{gathered}
H_{m}(\boldsymbol{\omega})=\sum_{i=0}^{|\boldsymbol{N}|-1} 2 E_{i}\left(\boldsymbol{N}^{T} \boldsymbol{\omega}-2 \pi \boldsymbol{b}\right)[\boldsymbol{C}]_{m i} \exp \left(-j \boldsymbol{\omega}^{T} \boldsymbol{n}_{i}\right), \\
m=0,1, \cdots,|\boldsymbol{M}|-1
\end{gathered}
$$




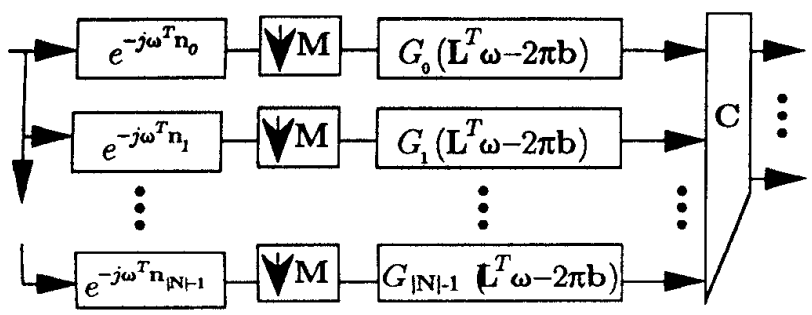

Fig. 18. Efficient implementation of the analysis bank of the two-parallelogram CMFB. The matrix $C$ is of dimension $|M|$ by $2|M|$.

where

$$
\begin{aligned}
{[\boldsymbol{C}]_{m i}=\cos \left(2 \pi\left(\boldsymbol{k}_{m+b \lambda_{0}}+\boldsymbol{b}\right)^{T} \boldsymbol{N}^{-1} \boldsymbol{n}_{i}\right), } & \\
m & =0,1, \cdots,|\boldsymbol{M}|-1, \quad i=0,1, \cdots,|\boldsymbol{N}|-1 .
\end{aligned}
$$

The above expression for the analysis filters gives rise to the efficient implementation in Fig. 18. The matrix $C$ is rectangular of dimension $|\boldsymbol{M}| \times|\boldsymbol{N}|$. The figure demonstrates that the complexity of the 2-D CMFB is that of the prototype plus $C$. The elements of $c$ are those of a nonseparable 2-D DCT.

Decomposition and Complexity of $C$ : We first define the the Kronecker product of two matrices $\boldsymbol{A}$ and $\boldsymbol{B}$ :

$$
\underbrace{\boldsymbol{A}}_{I \times K} \otimes \underbrace{\boldsymbol{B}}_{J \times L}=\underbrace{\left[\begin{array}{ccc}
a_{0,0} B & \cdots & a_{0, K-1} B \\
\vdots & \cdots & \vdots \\
a_{I-1,0} B & \cdots & a_{I-1, K-1} B
\end{array}\right]}_{I J \times K L} .
$$

The matrix $C$ can be decomposed as (to be shown in Appendix C)

$$
C=C_{1} \otimes C_{0}-S_{1} \otimes S_{0}
$$

where $C_{0}, S_{0}$ are $\lambda_{0} \times \lambda_{0}$ matrices, and $C_{1}, S_{1}$ are $\lambda_{1} \times 2 \lambda_{1}$ matrices given by

$$
\begin{aligned}
{\left[\boldsymbol{C}_{0}\right]_{k_{0} n_{0}}=\cos \left(\frac{2 \pi}{\lambda_{0}}\left(k_{0}-b_{0} / 2\right) n_{0}\right), } \\
{\left[\boldsymbol{S}_{0}\right]_{k_{0} n_{0}}=\sin \left(\frac{2 \pi}{\lambda_{0}}\left(k_{0}-b_{0} / 2\right) n_{0}\right), \quad 0 \leq k_{0}, n_{0}<\lambda_{0} } \\
{\left[\boldsymbol{C}_{1}\right]_{k_{1} n_{1}}=\cos \left(\frac{\pi}{\lambda_{1}}\left(k_{1}+0.5\right) n_{1}\right), } \\
{\left[S_{1}\right]_{k_{1} n_{1}}=\sin \left(\frac{\pi}{\lambda_{1}}\left(k_{1}+0.5\right) n_{1}\right), \quad 0 \leq k_{1}<\lambda_{1}, } \\
0 \leq n_{1}<2 \lambda_{1} .
\end{aligned}
$$

The implementation of $C$ is closely related to that of $\boldsymbol{C}_{0}, \boldsymbol{S}_{0}, \boldsymbol{C}_{1}$ and $\boldsymbol{S}_{1}$. The matrices $\boldsymbol{C}_{0}, \boldsymbol{S}_{0}, \boldsymbol{C}_{1}$ and $\boldsymbol{S}_{1}$ have elements resembling that of DCT and DST matrices. The complexity of these four matrices can be shown to be roughly $C\left(\lambda_{0}\right), C\left(\lambda_{0}\right), \frac{1}{2} C\left(2 \lambda_{1}\right)$ and $\frac{1}{2} C\left(2 \lambda_{1}\right)$. Using the decomposition in (19), we can further show that the complexity of the $C$ matrix is roughly equal to $4 \lambda_{1} C\left(\lambda_{0}\right)+\lambda_{0} C\left(2 \lambda_{1}\right)$. These properties of $C, C_{0}, S_{0}, C_{1}$ and $S_{1}$ will be verified in Appendix C.

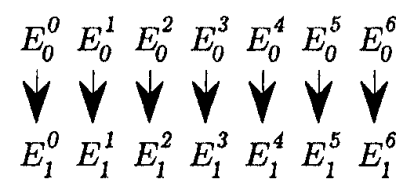

(a)

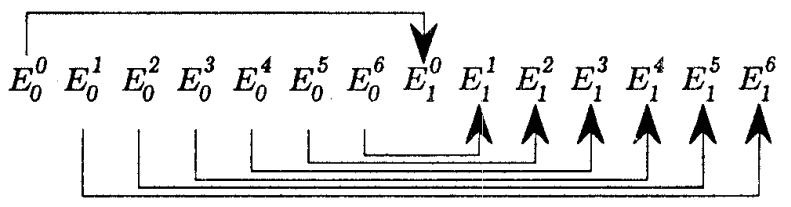

(b)

Fig. 19. Example 7.1. Two-parallelogram cosine modulated filter bank. (a) Polyphase components of $P(\omega)$ that are pairwise power complementary. (b) Polyphase components of $P(\omega)$ that are related due to linear phase of $P(\omega)$.

Example 7.1-Two-Parallelogram CMFB: Let

$$
\boldsymbol{M}=\left[\begin{array}{rr}
7 & -2 \\
0 & 1
\end{array}\right]=\left[\begin{array}{rr}
1 & -2 \\
0 & 1
\end{array}\right]\left[\begin{array}{ll}
7 & 0 \\
0 & 1
\end{array}\right] .
$$

Choose

$$
\boldsymbol{L}=\left[\begin{array}{ll}
1 & 0 \\
0 & 2
\end{array}\right]\left[\begin{array}{ll}
1 & 1 \\
1 & 2
\end{array}\right], \quad \text { then } \boldsymbol{N}=\left[\begin{array}{rr}
3 & -1 \\
2 & 4
\end{array}\right]
$$

With the above of choice of $L, L A T\left(\boldsymbol{L}^{T}\right)$ is quincunx. If we choose configuration $B$, then $l=\left[\begin{array}{ll}0 & 0.5\end{array}\right]^{T}$. Fig. 4 shows the supports of the analysis filters. By Theorem 6.1, the two-parallelogram CMFB has perfect reconstruction if the polyphase components of the prototype satisfy the power complementary condition given in (16). Fig. 19(a) shows the pairs of polyphase components that are power complementary. As the prototype is linear phase, the polyphase components are related in pairs. Fig. 19(b) shows these pairwise relations. In the figures, we use the notation $E_{n_{1}}^{n_{0}}(\boldsymbol{\omega})$ to denote the polyphase component $E_{n_{0}+n_{1} \lambda_{0}}(\boldsymbol{\omega})$. From the discussion for polyphase components in Section VI-C, if $\left(E_{0}^{i}(\omega), E_{1}^{i}(\omega)\right)$ for $i=1,2,3$, are power complementary pairs, then $\left(E_{0}^{i}(\boldsymbol{\omega}), E_{1}^{i}(\boldsymbol{\omega})\right)$ for $i=$ $4,5,6$, are guaranteed to be power complementary pairs. We can optimize $P(\omega)$ subject to only the condition that $E_{0}^{i}(\omega)$ and $E_{1}^{i}(\omega)$ for $i=1,2,3$, are power complementary. This condition can be satisfied by using the 2-D paraunitary lattice, [30].

Fig. 20 shows the support of impulse response of the prototype filter $p(\boldsymbol{n})$. The support of $p(\boldsymbol{n})$ resembles the shape of $S P D(2 N)$. Each solid dot represents a possibly nonzero coefficient of $p(\boldsymbol{n})$. In this optimization, each of the 14 polyphase components has four coefficients. The corresponding frequency response of the prototype is shown in Fig. 21. The stopband attenuation of the prototype is $17 \mathrm{~dB}$. The reason that the prototype cannot have good attenuation is due to the lack of vertex permissibility in two-parallelogram filter banks (Section III).

\section{Concluding Remarks}

In the application of subband coding, the support configuration of the analysis filters on a large scale determines the coding performance of the filter bank. In this paper, we 


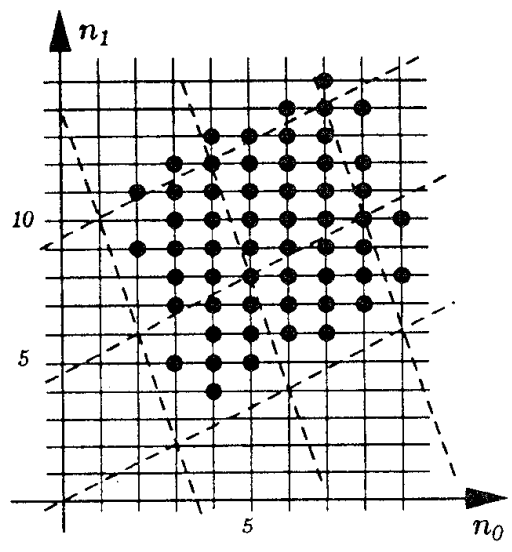

Fig. 20. Example 7.1. Two-parallelogram cosine modulated filter bank. The impulse response support of the prototype. Each solid dot represents a possibly nonzero coefficient of the prototype. (Intersection points of the dashed lines are on the lattice of $N$. Solid lines represent integers.)

have considered the class of two-parallelogram filter bank. The passband of each analysis filter consists of two parallelograms and the frequency plane is partitioned by parallelogram cells. (These types of configurations also have the potential application of extracting directional information-like edges.)

To facilitate the analysis, we introduced the notions of edge and vertex permissibility and explained that, although the former one is more important, both types of permissibility are necessary for good filter bank design. As elaborated upon in Section III, the two-parallelogram filter banks can possess edge or vertex permissibility but not both. As a consequence, the stopband attenuation of the filters in two-parallelogram perfect reconstruction filter banks cannot be arbitrary large. Extensions of the discussions of this paper for the case of linear-phase filters can be found in [19]

\section{APPENDIX A}

Summary of INTEGER MATRICES AND MultiRATE SyStems

\section{Fundamentals of Integer Matrices}

1) Unimodular Matrix: An integer matrix $U$ is unimodular if $|\boldsymbol{U}|=1$.

2) Notations $\mathcal{N}(\boldsymbol{M}),|\boldsymbol{M}|$ and $S P D(\boldsymbol{M})$ : Let $\boldsymbol{M}$ be a $D \times D$ nonsingular integer matrix. The notation $\mathcal{N}(\boldsymbol{M})$ is defined as the set of integer vectors of the form

$$
M \boldsymbol{x}, \quad \boldsymbol{x} \in[0,1)^{D} .
$$

The number of elements in $\mathcal{N}(\boldsymbol{M})$ is equal to $|\boldsymbol{M}|$, which is the absolute value of determinant of $M$. In the 1 D case, $D=1$, and $\mathcal{N}(\boldsymbol{M})=\{0,1,2, \cdots, \boldsymbol{M}-1\}$. The symmetric parallelepiped $S P D(\boldsymbol{M})$ is defined as $S P D(\boldsymbol{M})=\left\{\boldsymbol{M} \boldsymbol{x}, \boldsymbol{x} \in[-1,1)^{D}\right\}$.

3) Division Theorem for Integer Vectors: Let $M$ be a $D \times D$ matrix and $n$ be a $D \times 1$ integer vector. We can express $\boldsymbol{n}$ as $\boldsymbol{n}=\boldsymbol{n}_{0}+\boldsymbol{M} \boldsymbol{k}, \boldsymbol{n}_{0} \in \mathcal{N}(\boldsymbol{M})$. Moreover, $\boldsymbol{n}_{0}$ and $\boldsymbol{k}$ are unique. This relation is denoted by $\boldsymbol{n}=\boldsymbol{n}_{0} \bmod$ $M$. We will use the notation $n \stackrel{M}{=}$ if $n-k=M d$ for some integer vector $d$.

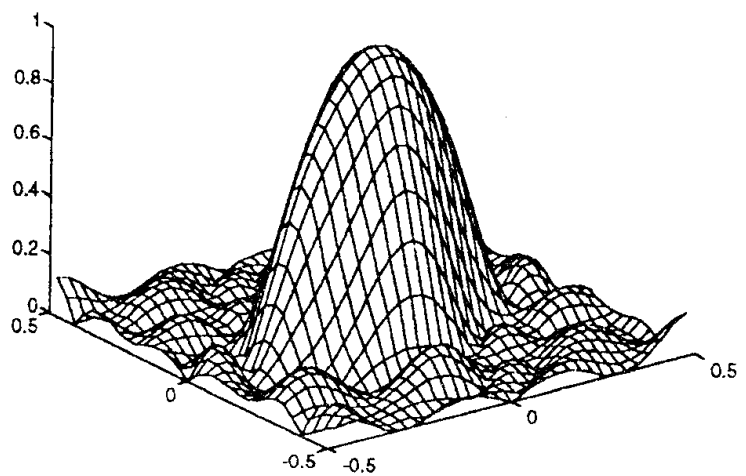

Fig. 21. Example 7.1. Two-parallelogram cosine modulated filter bank. The magnitude response of the prototype with frequency normalized by $2 \pi$.

4) Lattices: The lattice of a matrix $M$ is denoted by $\operatorname{LAT}(\boldsymbol{M})$. Two matrices $\boldsymbol{M}$ and $\boldsymbol{M}^{\prime}$ have the same lattice if and only if $M^{-1} M^{\prime}$ is a unimodular matrix. In this case, $\mathcal{N}(\boldsymbol{M})$ and $\mathcal{N}\left(\boldsymbol{M}^{\prime}\right)$ have the relation $\mathcal{N}(\boldsymbol{M})=\left\{\boldsymbol{n} \bmod \boldsymbol{M}, \boldsymbol{n} \in \mathcal{N}\left(\boldsymbol{M}^{\prime}\right)\right\}$. The lattice of a $2 \times 2$ matrix $\boldsymbol{M}$ is called quincunx if $\boldsymbol{M}=\left[\begin{array}{rr}1 & 1 \\ -1 & 1\end{array}\right] \boldsymbol{U}$ for some unimodular $U$.

5) Diagonalization of Integer Matrices: A $D \times D$ integer matrix $M$ can always be factorized as $\boldsymbol{M}=\boldsymbol{U} \boldsymbol{\Lambda} \boldsymbol{V}$, where $U$ and $V$ are unimodular integer matrices, and $\Lambda$ is a diagonal integer matrix.

\section{Preliminary of 2-D Multirate Systems}

1) Decimation Followed by Expansion: Given an $M$-fold decimator, the input $x(\boldsymbol{n})$ and the output $y(\boldsymbol{n})$ are related by $y(\boldsymbol{n})=x(\boldsymbol{M} \boldsymbol{n})$. In the frequency domain, the relation is

$$
Y(\boldsymbol{\omega})=\frac{1}{|\boldsymbol{M}|} \sum_{\boldsymbol{k} \in \mathcal{N}\left(\boldsymbol{M}^{T}\right)} X\left(\boldsymbol{M}^{-T}(\boldsymbol{\omega}-2 \pi \boldsymbol{k})\right) .
$$

Given an $\boldsymbol{M}$-fold expander, the input $x(\boldsymbol{n})$ and the output $y(\boldsymbol{n})$ are related by

$$
y(\boldsymbol{n})= \begin{cases}x\left(\boldsymbol{M}^{-1} \boldsymbol{n}\right), & \boldsymbol{n} \in L A T(\boldsymbol{M}) \\ 0, & \text { otherwise. }\end{cases}
$$

In the frequency domain, the relation is $Y(\omega)=$ $X\left(\boldsymbol{M}^{T} \boldsymbol{\omega}\right)$.

Consider the concatenation of the $M$-fold decimator followed by an $\boldsymbol{M}$-fold expander. The input $x(\boldsymbol{n})$ and the output $y(\boldsymbol{n})$ are related by

$$
y(\boldsymbol{n})= \begin{cases}x(\boldsymbol{n}), & \boldsymbol{n} \in \operatorname{LAT}(\boldsymbol{M}) \\ 0, & \text { otherwise. }\end{cases}
$$

In the frequency domain, the relation becomes

$$
Y(\boldsymbol{\omega})=\frac{1}{|\boldsymbol{M}|} \sum_{\boldsymbol{k} \in \mathcal{N}\left(\boldsymbol{M}^{T}\right)} X\left(\boldsymbol{\omega}-2 \pi \boldsymbol{M}^{-T} \boldsymbol{k}\right) .
$$

The output $Y(\boldsymbol{\omega})$ contains $X(\boldsymbol{\omega})$ and $|\boldsymbol{M}|-1$ images of $X(\omega)$, which are shifts of $X(\boldsymbol{\omega})$ by $2 \pi \boldsymbol{M}^{-T} \boldsymbol{k}$. 
2) Perfect Reconstruction 2-D Filter Bank: Consider the 2-D filter bank in Fig. 1. The output $\widehat{X}(\boldsymbol{\omega})$ is given by

$$
\widehat{X}(\boldsymbol{\omega})=\frac{1}{|\boldsymbol{M}|} \sum_{\boldsymbol{k} \in \mathcal{N}\left(\boldsymbol{M}^{T}\right)} A_{\boldsymbol{k}}(\boldsymbol{\omega}) X\left(\boldsymbol{\omega}-2 \pi \boldsymbol{M}^{-T} \boldsymbol{k}\right)
$$

where

$A_{\boldsymbol{k}}(\boldsymbol{\omega})=\sum_{m=0}^{|\boldsymbol{M}|-1} H_{m}\left(\boldsymbol{\omega}-2 \pi \boldsymbol{M}^{-T} \boldsymbol{k}\right) F_{m}(\boldsymbol{\omega}), \boldsymbol{k} \in \mathcal{N}\left(\boldsymbol{M}^{T}\right)$.

The filter bank is free from aliasing if the aliasing transfer function $A_{\boldsymbol{k}}(\boldsymbol{\omega})$ is 0 when $k \neq 0 \bmod \boldsymbol{M}^{T}$. The 2-D filter bank has perfect reconstruction if $\hat{X}(\omega)$ is a scaled and delayed version of $X(\omega)$. As in 1-D filter banks, the perfect reconstruction condition can be interpreted in terms of the polyphase matrices. Using polyphase decomposition, the analysis and synthesis filters have the form

$$
\begin{gathered}
H_{m}(\boldsymbol{\omega})=\sum_{\boldsymbol{k}_{i} \in \mathcal{N}(\boldsymbol{M})} E_{m, i}\left(\boldsymbol{M}^{T} \boldsymbol{\omega}\right) e^{-j \boldsymbol{\omega}^{T} \boldsymbol{k}_{i},} \\
m=0,1, \cdots,|\boldsymbol{M}|-1, \\
F_{m}(\boldsymbol{\omega})=\sum_{\boldsymbol{k}_{i} \in \mathcal{N}(\boldsymbol{M})} R_{i, m}\left(\boldsymbol{M}^{T} \boldsymbol{\omega}\right) e^{j \boldsymbol{\omega}^{T} \boldsymbol{k}_{i}} \\
m=0,1, \cdots,|\boldsymbol{M}|-1 .
\end{gathered}
$$

The $|\boldsymbol{M}| \times|\boldsymbol{M}|$ matrices $\boldsymbol{E}(\boldsymbol{\omega})$ and $\boldsymbol{R}(\boldsymbol{\omega})$ with $[\boldsymbol{E}(\boldsymbol{\omega})]_{m, i}=E_{m, i}(\boldsymbol{\omega})$ and $[\boldsymbol{R}(\boldsymbol{\omega})]_{m, i}=R_{m, i}(\boldsymbol{\omega})$ are, respectively, called the polyphase matrices for the analysis bank and the synthesis bank. The 2-D filter bank has perfect reconstruction if $\boldsymbol{R}(\boldsymbol{\omega}) \boldsymbol{E}(\boldsymbol{\omega})=\boldsymbol{I}_{\mid \boldsymbol{M}}$, where $\boldsymbol{I}_{\mid \boldsymbol{M}}$ denotes a $|\boldsymbol{M}| \times|\boldsymbol{M}|$ identity matrix.

\section{APPENDIX B}

PROOF OF THEOREM 6.1

The proof will be done in three steps. In the first step, we formulate the polyphase matrix $\boldsymbol{E}(\boldsymbol{\omega})$ of the analysis bank. As the 2-D CMFB is paraunitary, the filter bank has perfect reconstruction if and only if $\boldsymbol{E}^{\dagger}(\boldsymbol{\omega}) \boldsymbol{E}(\boldsymbol{\omega})$ is an identity matrix except some scalar. In the second step, we simplify the product $\boldsymbol{E}^{\dagger}(\boldsymbol{\omega}) \boldsymbol{E}(\boldsymbol{\omega})$ as much as possible without using the linear phase property of the prototype $P(\boldsymbol{\omega})$. As elaborated in Section VI-C, the polyphase components of the prototype are related in pairs due to the linear phase of $P(\boldsymbol{\omega})$. In the final step, we use those pairwise relations to show $\boldsymbol{E}^{\dagger}(\boldsymbol{\omega}) \boldsymbol{E}(\boldsymbol{\omega})=\boldsymbol{I}_{|\boldsymbol{M}|}$, provided that $E_{i}(\boldsymbol{\omega})$ are pairwise power complementary as in (16).

\section{Notations and Preliminaries for Appendix $B$}

For the convenience of derivation, we introduce the following notations:

1) The matrix $\boldsymbol{I}_{k}$ denotes a $k \times k$ identity matrix, and $\boldsymbol{J}_{k}$ denotes a $k \times k$ reversal matrix with nonzero entries $\left[J_{n, k-1-n}=1\right.$ for $n=0,1, \cdots, k-1$.

2) The DFT Matrix: The $\lambda \times \lambda$ DFT matrix $\omega_{\lambda}$ is given by $\left[\boldsymbol{W}_{\lambda}\right]_{m n}=W_{\lambda}^{m n}$, where $W_{\lambda}=e^{-j 2 \pi / \lambda}$.
3) Two properties of the Kronecker product (defined in Section VII) are relevant to our discussion.

(1) $(\boldsymbol{A} \otimes \boldsymbol{B})^{\dagger}=\boldsymbol{A}^{\dagger} \otimes \boldsymbol{B}^{\dagger}, \quad$ and

(2) $(\underbrace{A}_{I \times K} \otimes \underbrace{B}_{J \times L})(\underbrace{C}_{K \times M} \otimes \underbrace{D}_{L \times N})=(\underbrace{A C}_{I \times M}) \otimes(\underbrace{B D}_{J \times N})$.

Step 1-Polyphase Matrix $\boldsymbol{E}(\omega)$ of the Analysis Bank: Let $\boldsymbol{q}(\boldsymbol{\omega})$ be a $|\boldsymbol{N}| \times 1$ vector $\boldsymbol{q}(\boldsymbol{\omega})=\left[Q_{\boldsymbol{k}_{0}}(\boldsymbol{\omega})\right.$

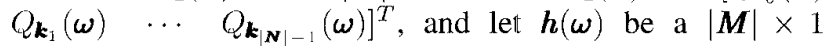
vector $\boldsymbol{h}(\boldsymbol{\omega})=\left[\begin{array}{llll}H_{0}(\boldsymbol{\omega}) & H_{1}(\boldsymbol{\omega}) & \cdots & H_{|\boldsymbol{M}|-1}(\boldsymbol{\omega})\end{array}\right]^{T}$, where $H_{i}(\omega)$ is as given in (9). In view of the discussion in Section $\mathrm{V}$, we have

$$
h(\omega)=G q(\omega)
$$

where

$$
\begin{aligned}
& \boldsymbol{G}=\left[\begin{array}{cccc}
\boldsymbol{I}_{b \lambda_{0}} & \boldsymbol{J}_{b} \otimes \boldsymbol{J}^{\prime} & \mathbf{0} & \mathbf{0} \\
\mathbf{0} & \mathbf{0} & \boldsymbol{I}_{\left(\lambda_{1}-b\right) \lambda_{0}} & \boldsymbol{J}_{\left(\lambda_{1}-b\right)} \otimes \boldsymbol{J}^{\prime}
\end{array}\right], \\
& \boldsymbol{J}^{\prime}=\left[\begin{array}{cc}
\boldsymbol{J}_{b_{0}+\mathbf{1}} & \mathbf{0} \\
\mathbf{0} & \boldsymbol{J}_{\lambda_{0}-b_{0}-1}
\end{array}\right]
\end{aligned}
$$

where the values of $b$ and $b_{0}$ are as given in Section V. We will first derive the matrix representation of $q(\omega)$ and, therefore, the matrix representation of $\boldsymbol{h}(\boldsymbol{\omega})$. From the expression of $\boldsymbol{h}(\boldsymbol{\omega})$, we can obtain the polyphase matrix $\boldsymbol{E}(\boldsymbol{\omega})$ of the analysis bank.

Using the polyphase representation of the prototype $P(\omega)$ in (14), we have

$$
\begin{aligned}
& Q_{\boldsymbol{k}_{m_{0}+\lambda_{0} m_{1}}}\left(\boldsymbol{\omega}+2 \pi \boldsymbol{N}^{-T} \boldsymbol{l}\right) \\
& =\sum_{i_{0}=0}^{\lambda_{0}-1} \sum_{i_{1}=0}^{2 \lambda_{1}-1} e^{j 2 \pi \boldsymbol{k}_{m_{0}+\lambda_{0} m_{1}}^{T} \boldsymbol{N}^{-1} \boldsymbol{n}_{i_{0}+\lambda_{0} i_{1}}} \\
& \cdot E_{i_{0}+\lambda_{0} i_{1}}\left(\boldsymbol{N}^{T} \boldsymbol{\omega}\right) e^{-j \boldsymbol{\omega}^{T} \boldsymbol{n}_{i_{0}+\lambda_{0} i_{1}}} \\
& \quad m_{0}=0,1, \cdots, \lambda_{0}-1 \\
& \quad m_{1}=0,1, \cdots, 2 \lambda_{1}-1
\end{aligned}
$$

By the definition of $\boldsymbol{k}_{m_{0}+\lambda_{0} m_{1}}$ and $\boldsymbol{n}_{i_{0}+\lambda_{0} i_{1}}$ given in (5) and (15), the term $e^{j 2 \pi \boldsymbol{k}_{m_{0}+\lambda_{0} m_{1}} \boldsymbol{N}^{-1} \boldsymbol{n}_{0_{0}+\lambda_{0} i_{1}}}$ reduces to $W_{\lambda_{0}}^{-m_{0} i_{0}} W_{2 \lambda_{1}}^{-m_{1} i_{1}}$, where $W_{\lambda}=e^{-j 2 \pi / \lambda}$. Then,

$$
\begin{aligned}
\boldsymbol{q}(\boldsymbol{\omega}+ & \left.2 \pi \boldsymbol{N}^{-T} \boldsymbol{l}\right) \\
= & \left(W_{2 \lambda_{1}}^{\dagger} \otimes W_{\lambda_{0}}^{\dagger}\right) \operatorname{diag}\left(E_{0}\left(\boldsymbol{N}^{T} \boldsymbol{\omega}\right), \cdots, E_{|\boldsymbol{N}|-1}\left(\boldsymbol{N}^{T} \boldsymbol{\omega}\right)\right) \\
& \cdot \boldsymbol{e}_{\boldsymbol{U} \boldsymbol{\Lambda}}(\boldsymbol{\omega})
\end{aligned}
$$

where $\operatorname{diag}\left(E_{0}\left(\boldsymbol{N}^{T} \boldsymbol{\omega}\right), \cdots, E_{|\boldsymbol{N}|-1}\left(\boldsymbol{N}^{T} \boldsymbol{\omega}\right)\right)$ is a $|\boldsymbol{N}| \times|\boldsymbol{N}|$ diagonal matrix with the $i$ th diagonal entry $E_{i}\left(N^{T} \omega\right)$. The matrices $\boldsymbol{W}_{\lambda_{0}}$ and $\boldsymbol{W}_{2 \lambda_{1}}$ are, respectively, $\lambda_{0} \times \lambda_{0}$ and $2 \lambda_{1} \times 2 \lambda_{1}$ DFT matrices. The vector $\boldsymbol{e}_{\boldsymbol{U} \boldsymbol{\Lambda}}(\boldsymbol{\omega})$ is the 2-D delay chain vector with respect to $\mathcal{N}(\boldsymbol{U} \boldsymbol{\Lambda}), \boldsymbol{e}_{\boldsymbol{U} \Lambda}(\boldsymbol{\omega})=$

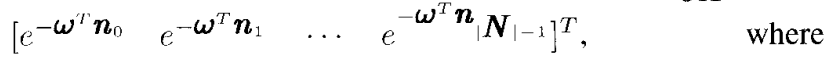
$\boldsymbol{n}_{i}$ is as given in (15). Since $\boldsymbol{n}_{i+|\boldsymbol{M}|}=\boldsymbol{n}_{i}+$ $U\left(\begin{array}{ll}0 & \lambda_{1}\end{array}\right)^{T}$, for $i=0,1, \cdots,|\boldsymbol{M}|-1,\left[\boldsymbol{e}_{\boldsymbol{U} \Lambda}(\boldsymbol{\omega})\right]_{i+\mid} \boldsymbol{M}_{\mid}=$ $\left.e^{-j \boldsymbol{\omega}^{T} \boldsymbol{U}_{(0}} \lambda_{1}\right)^{T}\left[\boldsymbol{e}_{\boldsymbol{U} \boldsymbol{\Lambda}}(\boldsymbol{\omega})\right]_{i}$. Let the 2-D delay chain with respect to $U \Lambda_{M}$ be

$$
\boldsymbol{e}_{\boldsymbol{U} \boldsymbol{\Lambda}_{M}}(\boldsymbol{\omega})=\left[\begin{array}{llll}
e^{-\boldsymbol{\omega}^{T} \boldsymbol{n}_{0}} & e^{-\boldsymbol{\omega}^{T} \boldsymbol{n}_{1}} & \cdots & e^{-\boldsymbol{\omega}^{T} \boldsymbol{n}_{|\boldsymbol{M}|-1}}
\end{array}\right]^{T} .
$$


Then, $\boldsymbol{c}_{\boldsymbol{U} \boldsymbol{\Lambda}}(\boldsymbol{\omega})=\left[\boldsymbol{e}_{\boldsymbol{U} \boldsymbol{\Lambda}_{\boldsymbol{M}}}^{T}(\boldsymbol{\omega}) e^{-j \boldsymbol{\omega}^{T} \boldsymbol{U}\left(0 \lambda_{1}\right)^{T}} \boldsymbol{e}_{\boldsymbol{U}}^{T} \boldsymbol{\Lambda}_{\boldsymbol{M}}(\boldsymbol{\omega})\right]^{T}$. Then, we have

$$
\begin{aligned}
\boldsymbol{h}(\boldsymbol{\omega}+ & \left.2 \pi \boldsymbol{N}^{-T} \boldsymbol{l}\right) \\
= & \underbrace{\boldsymbol{G}\left(\boldsymbol{W}_{2 \lambda_{1}}^{\dagger} \otimes \boldsymbol{W}_{\lambda_{0}}^{\dagger}\right)\left[\begin{array}{l}
\boldsymbol{E}_{0}\left(\boldsymbol{M}^{T} \boldsymbol{\omega}\right) \\
\boldsymbol{E}_{1}\left(\boldsymbol{M}^{T} \boldsymbol{\omega}\right)
\end{array}\right] \boldsymbol{G}_{0}}_{\boldsymbol{E}\left(\boldsymbol{M}^{T}\left(\boldsymbol{\omega}+2 \pi \boldsymbol{N}^{-T} \boldsymbol{l}\right)\right)} \\
& \cdot \boldsymbol{e}_{\boldsymbol{U} \boldsymbol{\Lambda}_{M}}\left(\boldsymbol{\omega}+2 \pi \boldsymbol{N}^{-T} \boldsymbol{l}\right)
\end{aligned}
$$

where $\boldsymbol{G}_{0}, \boldsymbol{E}_{0}(\boldsymbol{\omega})$ and $\boldsymbol{E}_{1}(\boldsymbol{\omega})$ are $|\boldsymbol{M}| \times|\boldsymbol{M}|$ diagonal matrices with

$$
\begin{aligned}
{\left[\boldsymbol{G}_{0}\right]_{i i} } & =e^{j 2 \pi \boldsymbol{l}^{T} \boldsymbol{N}^{-1} \boldsymbol{n}_{i}}, \quad\left[\boldsymbol{E}_{0}(\boldsymbol{\omega})\right]_{i i}=E_{i}\left(\boldsymbol{L}^{T} \boldsymbol{\omega}\right), \\
{\left[\boldsymbol{E}_{1}(\boldsymbol{\omega})\right]_{i i} } & =e^{-j \boldsymbol{\omega}^{T} \boldsymbol{V}_{\boldsymbol{M}}^{-1}(01)^{T}} E_{i+\lambda_{0} \lambda_{1}}\left(\boldsymbol{L}^{T} \boldsymbol{\omega}\right), \\
i & =0,1, \ldots,|\boldsymbol{M}|-1
\end{aligned}
$$

The matrix $E(\omega)$ as indicated in (20) is the polyphase matrix of the analysis bank. The 2-D CMFB is paraunitary if and only if $\boldsymbol{E}(\boldsymbol{\omega})$ is paraunitary. For simplicity, we will prove the paraunitariness of $\boldsymbol{E}\left(\boldsymbol{\omega}+2 \pi \boldsymbol{L}^{-T} \boldsymbol{l}\right)$, i.e., $\boldsymbol{E}^{\dagger}\left(\boldsymbol{\omega}+2 \pi \boldsymbol{L}^{-T} \boldsymbol{l}\right) \boldsymbol{E}(\boldsymbol{\omega}+$ $\left.2 \pi \boldsymbol{L}^{-T} \boldsymbol{l}\right)=\boldsymbol{I}_{|\boldsymbol{M}|}$.

Step 2 Simplification of the Product $\boldsymbol{E}^{\dagger}\left(\omega+2 \pi \boldsymbol{L}^{-T} \boldsymbol{l}\right)$ $\boldsymbol{E}\left(\boldsymbol{\omega}+2 \pi \boldsymbol{L}^{-T} \boldsymbol{l}\right)$ :

$$
\begin{aligned}
\boldsymbol{E}^{\dagger}(\boldsymbol{\omega}+ & \left.2 \pi \boldsymbol{L}^{-T} \boldsymbol{l}\right) \boldsymbol{E}\left(\boldsymbol{\omega}+2 \pi \boldsymbol{L}^{-T} \boldsymbol{l}\right) \\
= & \boldsymbol{G}_{0}^{\dagger}\left(\boldsymbol{E}_{0}^{\dagger}(\boldsymbol{\omega}) \boldsymbol{E}_{1}^{\dagger}(\boldsymbol{\omega})\right) \\
& \cdot \underbrace{\left(\boldsymbol{W}_{2 \lambda_{1}} \otimes \boldsymbol{W}_{\lambda_{0}}\right) \boldsymbol{G}^{T} \boldsymbol{G}\left(\boldsymbol{W}_{2 \lambda_{1}}^{\dagger} \otimes \boldsymbol{W}_{\lambda_{0}}^{\dagger}\right)}_{\boldsymbol{G}_{1}}\left[\begin{array}{l}
\boldsymbol{E}_{0}(\boldsymbol{\omega}) \\
\boldsymbol{E}_{1}(\boldsymbol{\omega})
\end{array}\right] \boldsymbol{G}_{0} .
\end{aligned}
$$

By the definition of $G$, we can verify that the product $G^{T} G$ is

$$
\boldsymbol{G}^{T} \boldsymbol{G}=\boldsymbol{I}_{|\boldsymbol{N}|}+\left[\begin{array}{cc}
\boldsymbol{J}_{2 b} & \mathbf{0} \\
\mathbf{0} & \boldsymbol{J}_{2\left(\lambda_{1}-b\right)}
\end{array}\right] \otimes \boldsymbol{J}^{\prime}
$$

It can be shown that the DFT matrix $W_{\lambda}$ has the following property:

$$
W_{\lambda}\left[\begin{array}{cc}
\boldsymbol{J}_{c} & 0 \\
0 & \boldsymbol{J}_{\lambda-c}
\end{array}\right] \boldsymbol{W}_{\lambda}^{\dagger}=\left[\begin{array}{cc}
1 & 0 \\
0 & \boldsymbol{J}_{\lambda-1}
\end{array}\right] \boldsymbol{\Gamma}_{\lambda}^{c-1}
$$

where $\boldsymbol{\Gamma}_{\lambda}$ is a $\lambda \times \lambda$ diagonal matrix with $\left[\boldsymbol{\Gamma}_{\lambda}\right]_{k k}=$ $e^{j 2 \pi k / \lambda}, k=0,1, \cdots, \lambda-1$. Using this relation and the second property of the Kronecker product, we have

$$
\begin{aligned}
\boldsymbol{G}_{1}= & \boldsymbol{I}_{|\boldsymbol{M}|}+\left\{\left[\begin{array}{cc}
1 & 0 \\
\mathbf{0} & \boldsymbol{J}_{2 \lambda_{1}-1}
\end{array}\right] \otimes\left[\begin{array}{cc}
1 & 0 \\
\mathbf{0} & \boldsymbol{J}_{\lambda_{0}-1}
\end{array}\right]\right\} \\
& \cdot\left(\boldsymbol{\Gamma}_{2 \lambda_{1}}^{b_{1}} \otimes \Gamma_{\lambda_{0}}^{b_{0}}\right) .
\end{aligned}
$$

It follows that

$$
\begin{aligned}
\boldsymbol{E}^{\dagger}(\boldsymbol{\omega} & \left.+2 \pi \boldsymbol{L}^{-T} \boldsymbol{l}\right) \boldsymbol{E}\left(\boldsymbol{\omega}+2 \pi \boldsymbol{L}^{-T} \boldsymbol{l}\right) \\
& =\boldsymbol{E}_{0}^{\dagger}(\boldsymbol{\omega}) \boldsymbol{E}_{0}(\boldsymbol{\omega})+\boldsymbol{E}_{1}^{\dagger}(\boldsymbol{\omega}) \boldsymbol{E}_{1}(\boldsymbol{\omega})+\boldsymbol{G}_{0}^{\dagger} \hat{\boldsymbol{G}}(\boldsymbol{\omega}) \boldsymbol{G}_{0}
\end{aligned}
$$

where

$$
\begin{aligned}
\hat{\boldsymbol{G}}(\boldsymbol{\omega})= & \left(\boldsymbol{E}_{0}^{\dagger}(\boldsymbol{\omega}) \boldsymbol{E}_{1}^{\dagger}(\boldsymbol{\omega})\right)\left\{\left[\begin{array}{cc}
1 & 0 \\
0 & \boldsymbol{J}_{2 \lambda_{1}-1}
\end{array}\right] \otimes\left[\begin{array}{cc}
1 & 0 \\
\mathbf{0} & \boldsymbol{J}_{\lambda_{0}-1}
\end{array}\right]\right\} \\
& \cdot\left(\Gamma_{2 \lambda_{1}}^{b_{1}} \otimes \Gamma_{\lambda_{0}}^{b_{0}}\right)\left[\begin{array}{l}
\boldsymbol{E}_{0}(\boldsymbol{\omega}) \\
\boldsymbol{E}_{1}(\boldsymbol{\omega})
\end{array}\right] .
\end{aligned}
$$

In the next step, $\hat{G}(\omega)$ will be shown to be equal to the $|\boldsymbol{M}| \times|\boldsymbol{M}|$ zero matrix. Since both $\boldsymbol{E}_{0}(\boldsymbol{\omega})$ and $\boldsymbol{E}_{1}(\boldsymbol{\omega})$ are both diagonal, $\boldsymbol{E}\left(\boldsymbol{\omega}+2 \pi L^{-T} \boldsymbol{l}\right)$ is paraunitary if and only if $\left[\boldsymbol{E}_{0}(\boldsymbol{\omega})\right]_{i i}$ and $\left[\boldsymbol{E}_{1}(\boldsymbol{\omega})\right]_{i i}$ are power complementary. Equivalently, $\left(E_{i}(\boldsymbol{\omega}), E_{i+\lambda_{0} \lambda_{1}}(\boldsymbol{\omega})\right)$ is a power complementary pair in 2-D sense.

Step 3-Proof of $\hat{\boldsymbol{G}}(\boldsymbol{\omega})=0$ : As mentioned in Section V, $b_{1}$ is odd. Therefore, $\left[\boldsymbol{I}_{2 \lambda}^{b_{1}}\right]_{k+\lambda_{1}, k+\lambda_{1}}=-\left[\Gamma_{2 \lambda}^{b_{1}}\right]_{k k}$ for $k=$ $0,1, \cdots, \lambda_{1}-1$, and hence, $\left[\Gamma_{2 \lambda_{1}}^{b_{1}} \otimes \Gamma_{\lambda_{0}}^{b_{0}}\right] k+\lambda_{0} \lambda_{1}, k+\lambda_{0} \lambda_{1}=$ $-\left[\Gamma_{2 \lambda_{1}}^{b_{1}} \Gamma_{\lambda_{0}}^{b_{0}}\right]_{k k}, k=0,1, \cdots, \lambda_{0} \lambda_{1}-1$. Let $\Gamma_{2 \lambda_{1}}^{b_{1}} \otimes \Gamma_{\lambda_{0}}^{b_{0}}=$ $\left[\begin{array}{cc}\Gamma & 0 \\ 0 & -\Gamma\end{array}\right]$. We observe that

$$
\left(\boldsymbol{\Gamma}_{2 \lambda_{1}}^{b_{1}} \otimes \boldsymbol{\Gamma}_{\lambda_{0}}^{b_{0}}\right)\left[\begin{array}{l}
\boldsymbol{E}_{0}(\boldsymbol{\omega}) \\
\boldsymbol{E}_{1}(\boldsymbol{\omega})
\end{array}\right]=\left[\begin{array}{r}
\boldsymbol{E}_{0}(\boldsymbol{\omega}) \\
-\boldsymbol{E}_{1}(\boldsymbol{\omega})
\end{array}\right] \boldsymbol{\Gamma} .
$$

Consider the partition $\left[\begin{array}{cc}1 & 0 \\ 0 & J_{2 \lambda_{1}-1}\end{array}\right]=\left[\begin{array}{ll}A & B \\ B & A\end{array}\right]$, then $A=$

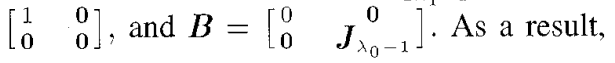

$$
\begin{aligned}
\hat{\boldsymbol{G}}(\boldsymbol{\omega}) \boldsymbol{I}^{\dagger} & =\underbrace{\boldsymbol{E}_{0}^{\dagger}(\boldsymbol{\omega})(\boldsymbol{A} \otimes \hat{\boldsymbol{J}}) \boldsymbol{E}_{0}(\boldsymbol{\omega})-\boldsymbol{E}_{1}^{\dagger}(\boldsymbol{\omega})(\boldsymbol{A} \otimes \hat{\boldsymbol{J}}) \boldsymbol{E}_{1}(\boldsymbol{\omega})}_{\hat{G}_{1}(\boldsymbol{\omega})} \\
& +\underbrace{\boldsymbol{E}_{1}^{\dagger}(\boldsymbol{\omega})(\boldsymbol{B} \otimes \hat{\boldsymbol{J}}) \boldsymbol{E}_{0}(\boldsymbol{\omega})-\boldsymbol{E}_{0}^{\dagger}(\boldsymbol{\omega})(\boldsymbol{B} \otimes \hat{\boldsymbol{J}}) \boldsymbol{E}_{1}(\boldsymbol{\omega})}_{\hat{G}_{2}(\boldsymbol{\omega})}
\end{aligned}
$$

where $\hat{J}=\left[\begin{array}{ll}1 & 0 \\ 0 & J_{\lambda_{0}-1}^{0}\end{array}\right]$.

By the definition of $\boldsymbol{A}$ and $\hat{J}_{0}$, we can verify that $\hat{\boldsymbol{G}}_{1}(\boldsymbol{\omega})=0$ if

$$
\begin{aligned}
E_{0}^{*}(\boldsymbol{\omega}) E_{0}(\boldsymbol{\omega}) & =E_{\lambda_{0} \lambda_{1}}^{*}(\boldsymbol{\omega}) E_{\lambda_{0} \lambda_{1}}(\boldsymbol{\omega}), \\
E_{i_{0}}^{*}(\boldsymbol{\omega}) E_{\lambda_{0}-i_{0}}(\boldsymbol{\omega}) & =E_{i_{0}+\lambda_{0} \lambda_{1}}^{*}(\boldsymbol{\omega}) E_{\lambda_{0}-i_{0}+\lambda_{0} \lambda_{1}}(\boldsymbol{\omega}), \\
& 0<i_{0}<\lambda_{0} .
\end{aligned}
$$

Similarly, $\hat{\boldsymbol{G}}_{2}(\boldsymbol{\omega})=0$ if

$$
\begin{gathered}
e^{j \boldsymbol{\omega}^{T} \boldsymbol{V}^{-1}(01)^{T}} E_{\left(i_{1}+\lambda_{1}\right) \lambda_{0}}^{*}(\boldsymbol{\omega}) E_{\left(\lambda_{1}-i_{1}\right) \lambda_{0}}(\boldsymbol{\omega}) \\
=E_{i_{1} \lambda_{0}}^{*}(\boldsymbol{\omega}) E_{\left(2 \lambda_{1}-i_{1}\right) \lambda_{0}}(\boldsymbol{\omega}), \quad 0<i_{1}<\lambda_{1}, \\
e^{j \boldsymbol{\omega}^{T} \boldsymbol{V}^{-1}(01)^{T}} E_{i_{0}+\left(i_{1}+\lambda_{1}\right) \lambda_{0}}^{*}(\boldsymbol{\omega}) E_{\lambda_{0}-i_{0}+\left(\lambda_{1}-i_{1}\right) \lambda_{0}}(\boldsymbol{\omega}) \\
=E_{i_{0}+i_{1} \lambda_{0}}^{*}(\boldsymbol{\omega}) E_{\lambda_{0}-i_{0}+\left(2 \lambda_{1}-i_{1}\right) \lambda_{0}}(\boldsymbol{\omega}), \\
\quad 0<i_{0}<\lambda_{0}, \quad 0<i_{1}<\lambda_{1} .
\end{gathered}
$$

Equations (21) and (22) can be verified by using the the relation of the polyphase components in (17).

APPENDIX C

DeCOMPOSITION AND COMPLEXITY OF THE MATRIX $C$

1) Decomposition of the Matrix $C$ : Substitute in $k_{k_{0}+\lambda_{0} k_{1}}$ $=V^{T}\left(\begin{array}{ll}k_{0} & k_{1}\end{array}\right)^{T}$, and $n_{n_{0}+\lambda_{0} n_{1}}=V^{T}\left(\begin{array}{ll}n_{0} & n_{1}\end{array}\right)^{T}$, and we have

$[C]_{k_{0}+\lambda_{0} k_{1}, n_{0}+\lambda_{0} n_{1}}$

or

$$
=\cos \left(\frac{2 \pi}{\lambda_{0}}\left(k_{0}-b_{0} / 2\right) n_{0}+\frac{\pi}{\lambda_{1}}\left(k_{1}+0.5\right) n_{1}\right)
$$

$[C]_{k_{0}+\lambda_{0} k_{1}, n_{0}+\lambda_{0} n_{1}}=\left[\boldsymbol{C}_{1}\right]_{k_{1} n_{1}}\left[\boldsymbol{C}_{0}\right]_{k_{0} n_{0}}-\left[\boldsymbol{S}_{1}\right]_{k_{1} n_{1}}\left[\boldsymbol{S}_{\mathbf{0}}\right]_{k_{0} n_{0}}$.

Then, we have $C=C_{1} \otimes C_{0}-S_{1} \otimes S_{0}$. 
2) The Complexity of the Matrices $C_{0}, S_{0}, C_{1}$ and $S_{1}$ : The DCT and DST matrices are categorized into four types in [31]. The matrix $C_{1}$ has the first $\lambda_{1}$ rows of a $2 \lambda_{1} \times 2 \lambda_{1}$ type II DCT. In some implementation methods, $C_{1}$ requires only half the computation of a $2 \lambda_{1}$ point DCT. Likewise, $S_{1}$ is the upper half of a $2 \lambda_{1} \times 2 \lambda_{1}$ type II DST and needs half the complexity of a $2 \lambda_{1}$ point DST. When $b_{0}$ is odd, $C_{0}$ is a rearrangement of a type II DCT matrix. Computation of $C_{0}$ is equivalent to that of a $\lambda_{0}$ point DCT. Similarly, $\boldsymbol{S}_{0}$ can be obtained by rearranging a type II DST matrix, and the computation of $\boldsymbol{S}_{0}$ is equivalent to that of a $\lambda_{0}$-point DST. However, when $b_{0}$ is even, $C_{0}$ and $S_{0}$ become, respectively, rearrangement of type I DCT and type I DST. Complexity of $C_{0}$ and $S_{0}$ are comparable with that of a $\lambda_{0}+1$ point type I DCT and $\lambda_{0}-1$ point type I DST.

3) The Complexity of the Matrix $C$ : The matrix $C$ has two parts, namely, $\boldsymbol{C}_{1} \otimes \boldsymbol{C}_{0}$ and $\boldsymbol{S}_{1} \otimes \boldsymbol{S}_{0}$. We will look into details of computing $C_{1} \otimes C_{0}$. The implementation of $\boldsymbol{S}_{1} \otimes \boldsymbol{S}_{0}$ is similar. Suppose the input of $\boldsymbol{C}$ is $\boldsymbol{x}$, which is a $|\boldsymbol{N}| \times 1$ vector. Let $\boldsymbol{y}=\left(\boldsymbol{C}_{1} \otimes \boldsymbol{C}_{0}\right) \boldsymbol{x}$, which is a $|\boldsymbol{M}| \times 1$ vector. By the property of the Kronecker product, we can also write $\boldsymbol{C}_{1} \otimes \boldsymbol{C}_{0}$ as $\left(\boldsymbol{C}_{1} \otimes \boldsymbol{I}_{\lambda_{0}}\right)\left(\boldsymbol{I}_{2 \lambda_{1}} \otimes \boldsymbol{C}_{0}\right)$. The vector $\boldsymbol{y}$ can be obtained in two steps: i) computation of $I_{2 \lambda_{1}} \otimes C_{0}$ and ii) computation of $C_{1} \otimes I_{\lambda_{0}}$. We analyze these two operations as follows: i) Partition $x$ into $2 \lambda_{1}$ vectors, each of size $\lambda_{0}$, i.e., $\boldsymbol{x}=\left[\begin{array}{llll}x_{0}^{T} & x_{1}^{T} & \cdots & x_{2 \lambda_{1}-1}^{T}\end{array}\right]^{T}$. Let $\boldsymbol{w}=\left(\boldsymbol{I}_{2 \lambda_{1}} \otimes C_{0}\right) \boldsymbol{x}$ be the output of the first step, and then, $\boldsymbol{w}=\left[\begin{array}{llll}\left(\boldsymbol{C}_{0} \boldsymbol{x}_{0}\right)^{T} & \left(\boldsymbol{C}_{0} \boldsymbol{x}_{1}\right)^{T} & \cdots & \left(\boldsymbol{C}_{0} \boldsymbol{x}_{2 \lambda_{1}-1}\right)^{T}\end{array}\right]^{T}$. The computation of $\boldsymbol{w}$ requires $2 \lambda_{1} C\left(\lambda_{0}\right)$. ii) It can be verified that after some row exchanges and column exchanges $C_{1} \otimes I_{\lambda_{0}}$ assumes the form $I_{\lambda_{0}} \otimes C_{1}$, namely, $\boldsymbol{C}_{1} \otimes \boldsymbol{I}_{\lambda_{0}}=\boldsymbol{P}_{1}\left(\boldsymbol{I}_{\lambda_{0}} \otimes \boldsymbol{C}_{1}\right) \boldsymbol{P}_{2}$, where $\boldsymbol{P}_{1}$ and $\boldsymbol{P}_{2}$ are permutation matrices. Partition $\boldsymbol{P}_{2} \boldsymbol{w}$ into $\lambda_{0}$ vectors $\boldsymbol{P}_{2} \boldsymbol{w}=$ $\left[\begin{array}{llll}\boldsymbol{w}_{0}^{T} & \boldsymbol{w}_{1}^{T} & \cdots & \boldsymbol{w}_{\lambda_{0}-1}^{T}\end{array}\right]^{T}$. Each of $\boldsymbol{w}_{i}^{\prime} g$ is a $2 \lambda_{1} \times 1$ vector. Then, $\left.\boldsymbol{y}=\boldsymbol{P}_{\mathbf{1}}\left[\begin{array}{llll}\left(\boldsymbol{C}_{1} \boldsymbol{w}_{0}\right)^{T} & \left(\boldsymbol{C}_{1} \boldsymbol{w}_{1}\right)^{T} & \cdots & \left(\boldsymbol{C}_{1} \boldsymbol{w}_{\lambda_{0}-1}\right.\end{array}\right)^{T}\right]^{T}$. As $\boldsymbol{P}_{1}$ and $\boldsymbol{P}_{2}$ are permutation matrices and require no computation, operation $\boldsymbol{C}_{1} \otimes \boldsymbol{I}_{\lambda_{0}}$ can be completed with complexity $\left(\lambda_{0} / 2\right) C\left(2 \lambda_{1}\right)$.

Therefore, the computation needed for $C_{1} \otimes C_{0}$ is $2 \lambda_{1} C\left(\lambda_{0}\right)+\left(\lambda_{0} / 2\right) C\left(2 \lambda_{1}\right)$. The matrix $S_{1} \otimes S_{0}$ has the same complexity. This verifies that complexity of $C$ is $4 \lambda_{1} C\left(\lambda_{0}\right)+\lambda_{0} C\left(2 \lambda_{1}\right)$.

\section{REFERENCES}

[1] T. Chen and P. P. Vaidyanathan, "Consideration in multidimensional filter bank design," in Proc. Int. Symp. Circuits Syst, May 1993.

[2] P. P. Vaidyanathan, Multirate Systems and Filter Banks. Englewood Cliffs, NJ: Prentice-Hall, 1993.

[3] M. Vetterli, "Multidimensional subband coding : some theory and algorithms," Signal Processing, vol. 6, pp. 97-112, Apr. 1984.

[4] J. W. Woods and S. D. O'Neil, "Subband coding of images," IEEE Trans. Accoust., Speech Signal Processing, vol. ASSP-34, pp. 1278-1288, Oct. 1986.

[5] R. Ansari and C. Guillemot, "Exact reconstruction filter banks using diamond FIR filters." in Proc. Int. Conf. New Trends Comm. Contr. Signal Processing. Turkey, July 1990.

[6] E. Viscito and J. P. Allebach, "The analysis and design of multidimensional FIR perfect reconstruction filter banks for arbitrary sampling lattices," IEEE Trans. Circuits Syst., vol. 38, pp. 29-41, Jan. 1991.

[7] R. H. Bamberger and M. J. T. Smith, "A filter bank for the directional decomposition of images: theory and design," IEEE Trans. Signal Processing, vol. 40, Apr. 1992.
[8] I. A. Shah and A. A. C. Kalker, "Generalized theory of multidimensional $M$-band filter bank design," EUSIPCO, pp. 969-972, 1992.

[9] S. C. Chan, "Two dimensional nonseparable modulated filter banks," in Proc. Int. Conf. Acoust., Speech, Signal Processing, Apr. 1994.

[10] J. Kovacevic, "Local cosine bases in two dimensions," in Proc. Int. Conf. Acoust, Speech, Signal Processing, vol. IV, May 1995, pp. 2125-2128.

[11] X.-G. Xia and B. W. Suter, "A family of 2-dimensional nonseparable Malvar wavelets," Applied Comput. Harmonic Anal, vol. 2, pp. 243-256, July 1995 .

[12] __ . "Construction of Malvar wavelets on hexagons," Applied Comput. Harmonic Anal., vol. 3, pp. 65-71, Jan. 1996.

[13] _ " "A systematic construction method for spatial-varying FIR filter banks with perfect reconstruction," in Proc. Int. Conf. Image Processing, Austin, TX, Nov. 1994

[14] M. Ikehara, "Cosine-modulated 2 dimensional FIR filter banks satisfying perfect reconstruction," in Proc. Int. Conf. Acoust., Speech. Signal Processing, Apr. 1994.

[15] M. Ikehara, "Modulated 2 dimensional perfect reconstruction FIR filter banks with permissible passbands," in Proc. Int. Conf. Acoust., Speech, Signal Processing, May 1995, pp. 1468-1471.

[16] Y.-P. Lin and P. P. Vaidyanathan, "Two-dimensional paraunitary cosine modulated perfect reconstruction filter banks," in Proc. Int. Symp. Circuits Syst., Apr. 1995, pp. 752-755.

[17] , "On the study of four parallelogram filter banks," submitted to IEEE Trans. Signal Processing, Oct. 1995.

[18] __ "Two-dimensional four-parallelogram filter banks," in Proc. 29th Ann. Asilomar Conf. Signals, Syst. Comput., Oct. 1995.

[19] _ "Two-dimensional linear-phase cosine modulated filter banks," in Proc. Int. Symp. Circuits Syst., May 1996.

[20] V. Sathe and P. P. Vaidyanathan, "Effects of multirate systems on the statistical properties of random inputs," IEEE Trans. Signal Processing, vol. 41, no. 1, pp. 131-146, Jan. 1993.

[21] T. Chen and P. P. Vaidyanathan, "Recent developments in multidimensional multirate systems," IEEE Trans. Circuits Syst. Video Technol., vol. 3, Apr. 1993.

[22] A. V. Oppenheim, A. S. Willsky, and I. Young, Signals and Systems. Englewood Cliffs, NJ: Prentice-Hall, 1983.

[23] H. J. Nussbaumer, "Pseudo QMF filter bank," IBM Tech. Disclosure Bull., vol. 24, pp. 3081-3087, Nov. 1981

[24] J. H. Rothweiler, "Polyphase quadrature filters, a new subband coding technique," in Proc. IEEE Int. Conf. Acoust., Speech Signal Processing, Apr. 1983, pp. 1980-1983.

[25] P. L. Chu, "Quadrature mirror filter design for an arbitrary number of equal bandwidth channels," IEEE Trans. Acoustic, Speech Signal Processing, vol. 33, no. 2, pp. 203-218, Feb. 1985.

[26] T. A. Ramstad, "Cosine modulated analysis-synthesis filter bank with critical sampling and perfect reconstruction," in Proc. IEEE Int. Conf. Acoustic, Speech Signal Processing, Toronto, Canada, May 1991, pp. 1789-1792.

[27] H. S. Malvar, Signal Processing with Lapped Transforms. Norwood, MA: Artcch House, 1992.

[28] R. D. Koilpillai and P. P. Vaidyanathan, "Cosine-modulated FIR filter banks satisfying perfect reconstruction," IEEE Trans. Signal Processing, vol. 40, pp. $770-783$, Apr. 1992.

[29] Y. Lin and P. P. Vaidyanathan, "Sampling theorems of two-dimensional signals." in Proc. Int. Conf. Acoust. Speech, Signal Processing, Apr. 1996.

[30] V. C. Liu and P. P. Vaidyanathan, "On factorization of subclass of 2D digital FIR lossless matrices for 2-D QMF bank application," IEEE Trans. Circuits Syst., June 1990.

131] P. Yip and K. R. Rao, "Fast discrete transforms," in Handbook of Digital Signal Processing, D. F. Elliott, Ed. San Diego, CA: Academic, 1987.

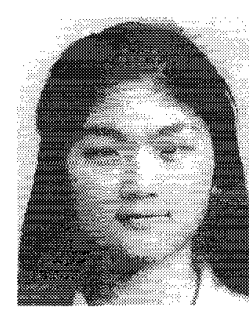

Yuan-Pei Lin (S'93) was born in Taipci, Taiwan, 1970. She received the B.S. degree in control engineering from the National Chiao-Tong University, Hsinchu, Taiwan, in 1992 and the M.S. degree in electrical engineering from the California Institute of Technology, Pasadena, in 1993. She is currently pursuing the doctoral degree at the California Institute of Technology.

Her research interests include multirate filter banks, wavelets, and multidimensional signal processing 


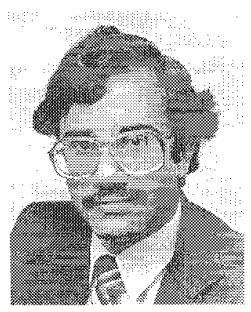

P. P. Vaidyanathan (S'80-M'83-SM'88-F'91) was born in Calcutta, India, on October 16, 1954. He received the B.Sc. (Hons.) degree in physics and the B.Tech. and M.Tech. degrees in radiophysics and electronics, all from the University of Calcutta, India, in 1974, 1977, and 1979, respectively. He received the $\mathrm{Ph} . \mathrm{D}$ degrec in electrical and computer engineering from the University of California at Santa Barbara in 1982. He was a post-doctoral fellow at the University of California, Santa Barbara, from September 1982 to March 1983.

In March 1983, he joined the electrical engineering department of the Calfornia Institute of Technology, Pasadena, as an Assistant Professor, and since 1993, he has been Professor of electrical engineering there. His main research interests are in digital signal processing, multirate systems, wavelet transforms, and adaptive filtering. He has authored a number of papers in IEEE journals and is the author of the book entitled Multirate Systems and Filter Banks. He has written several chapters for various signal processing handbooks.

Dr. Vaidyanathan served as Vice Chairman of the Technical Program committee for the 1983 IEEE International Symposium on Circuits and Systems and as the Technical Program Chairman for the 1992 IEEE International Symposium on Circuits and Systems. He was an Associate Editor for the IEEE TRAnsactions on CiRCUITS AND Systems for the period 1985 to 1987 and is currently an Associate Editor for the IEEE SiGNAL PROCESSING LETTERS and a consulting editor for the journal Applied and Computational Harmonic Analysis. He was a recipient of the Award for Excellence in Teaching at the California Institute of Technology for the years 1983 to 19841992 to 1993 , and 1993 to 1994 . He also received the NSF's Presidential Young Investigator award in 1986. In 1989, he received the IEEE ASSP Senior Award for his paper on multirate perfect-reconstruction filter banks. In 1990, he was the recipient of the S. K. Mitra Memorial Award from the Institute of Electronics and Telecommuncations Engineers, India, for his joint paper in the IETE journal. He was also the coauthor of a paper on linear-phase perfect reconstruction filter banks in the IEEE SP Transactions for which the first author (T. Nguyen) received the Young Outstanding Author award in 1993. He received the 1995 F. E. Terman Award of the American Society for Engineering Education, which was sponsored by Hewlett Packard $\mathrm{Co}$. He has been chosen a distinguished lecturer for the IEEE Signal Processing Society for the year 1996 to 1997. 